\title{
Orientations, semiorders, arrangements, and parking functions
}

\author{
Sam Hopkins \\ Department of Mathematics \\ Reed College \\ Portland, OR, U.S.A. \\ hopkins.reed.edu
}

\author{
David Perkinson \\ Department of Mathematics \\ Reed College \\ Portland, OR, U.S.A \\ davidp@reed.edu
}

Submitted: Jan 15, 2012; Accepted: Sep 3, 2012; Published: Oct 25, 2012

Mathematics Subject Classifications: 52C35, $05 \mathrm{C} 25$

\begin{abstract}
It is known that the Pak-Stanley labeling of the Shi hyperplane arrangement provides a bijection between the regions of the arrangement and parking functions. For any graph $G$, we define the $G$-semiorder arrangement and show that the PakStanley labeling of its regions produces all $G$-parking functions.
\end{abstract}

In his study of Kazhdan-Lusztig cells of the affine Weyl group of type $A_{n-1}$, [15], J.-Y. Shi introduced the arrangement of hyperplanes in $\mathbb{R}^{n}$ now known as the Shi arrangement:

$$
x_{i}-x_{j}=0,1 \quad 1 \leqslant i<j \leqslant n .
$$

Among other things, he proved that the number of regions in the complement of this set of hyperplanes is $(n+1)^{(n-1)}$, Cayley's formula for the number of trees on $n+1$ labeled vertices. The first bijective proof of this fact is due to Pak and Stanley, [16], who provide a method for labeling the regions with parking functions of size $n$. Given a graph $G$, Postnikov and Shapiro, [14], introduced the notion of a $G$-parking function. In the case where there exists a vertex $q$ connected by edges to every other vertex of $G$, Duval, Klivans, and Martin, [7], have defined the G-Shi arrangement, and conjecture that when its regions are labeled by the method of Pak and Stanley, the resulting labels are exactly the $G$-parking functions with respect to $q$. In this case, however, there may be duplicates among the labels. Letting $G$ be the complete graph on $n+1$ vertices recaptures the original result of Pak and Stanley. Our work was motivated by this conjecture.

Figure 1 serves as a guide to our paper. The four corners of the square in Figure 1 are labeled by structures we associate to a graph $G$, which we now describe.

Parking functions and superstable configurations. Chip-firing is a key tool for us. In the abelian sandpile model for $G$, one first chooses a vertex of $G$ to serve as the 


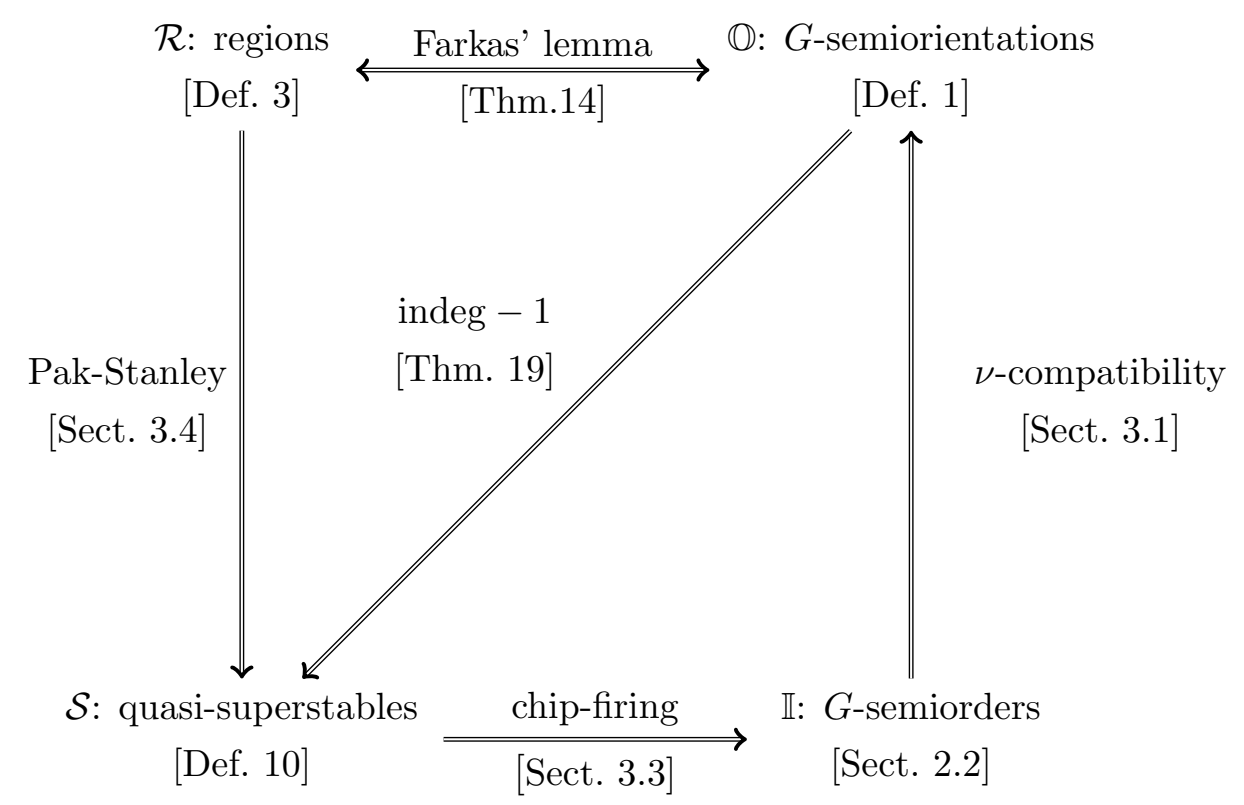

Figure 1: Schematic diagram of results.

"sink." Then placing grains of sand (or chips) on each of the nonsink vertices defines a configuration, $c$, on $G$. A vertex $v$ is unstable in $c$ if it has at least as many grains of sand as its degree. In that case, one may fire (or topple) $v$ by sending one grain of sand from $v$ to each of its neighbors. In this process neighbors of $v$ may become unstable, themselves. Sand is not necessarily conserved under firing: grains of sand that are sent to the sink vertex disappear. For undirected, connected graphs - to which we limit ourselves here by repeatedly firing vertices, $c$ is eventually transformed into a stable configuration, i.e., one with no unstable vertices.

Instead of firing one vertex at a time, one may consider a firing rule that allows sets of vertices to be fired simultaneously. This gives rise to a stronger type of stability and a corresponding set of superstable configurations (see Section 2.4). These superstable configurations serve to define $G$-parking functions. A $G$-parking function with respect to the chosen sink is a function from the vertices of $G$ to the integers whose value at the sink is -1 and whose values at the nonsink vertices are the numbers of grains of sand for some superstable configuration on $G$. Thus, there is only a slight difference between superstable configurations and $G$-parking functions, and at any rate, they are in oneto-one correspondence. Parking functions are more widely-known, and they originally arose independently (for instance in [11, p. 545]). This accounts for why they, and not superstable configurations, are mentioned in the title of this paper. However, given the importance of chip-firing to our work, we will mainly refer to superstable configurations outside of this introduction.

Although $G$-parking functions and superstable configurations are defined with respect to a chosen sink vertex, we have found it natural and convenient to first prove our results in a sinkless context, akin to working in projective space rather than affine space. To this 
end, we define generalized $G$-parking functions, which we call quasi-superstable divisors on $G$. These appear in the bottom left corner of Figure 1, denoted by $\mathcal{S}$. As indicated by the figure, quasi-superstables are formally defined in Definition 10.

Semiorders and the semiorder arrangement. The semiorder arrangement, [17], is the set of $n(n-1)$ hyperplanes in $\mathbb{R}^{n}$ given by

$$
x_{i}-x_{j}=1, \quad i, j \in\{1, \ldots, n\}, i \neq j .
$$

Its regions are in bijection with certain $n$-element posets called semiorders.

A $G$-semiorder is a semiorder whose elements are the vertices of $G$. The collection of $G$-semiorders, denoted by $\mathbb{I}$, appears in the bottom right corner of Figure 1 . In the same way that a Shi arrangement is modified by Duval, Klivans, and Martin in [7] to take into account the structure of a graph, we modify a semiorder arrangement to produce the $G$ semiorder arrangement associated with $G$. The regions of the $G$-semiorder arrangement are denoted by $\mathcal{R}$ in the top left corner of Figure 1.

Fixing a sink vertex, $v$, we then refine the definition of a $G$-semiorder arrangement to get the $(G, v)$-semiorder arrangement (Definition 28).

Orientations. A partial orientation of $G$ consists of orienting some, not necessarily all, of the edges of $G$. The $G$-semiorientations, denoted by $\mathbb{O}$ in the top right of Figure 1, are the partial orientations satisfying an extra condition relative to the cycles of the underlying graph, $G$.

Relations among the structures. Using the method of Pak and Stanley, we label each region of the $(G, v)$-semiorder arrangement with what amounts to a function from the vertices of $G$ to the integers taking the value -1 at $v$. The main goal of this paper is Theorem 32, which, along with Theorem 34, shows that the set of Pak-Stanley labels that are negative only at the sink are exactly the set of $G$-parking functions. The set of Pak-Stanley labels consists of only $G$-parking functions exactly when the sink is adjacent to every nonsink vertex.

Our main goal, just described, is a consequence of the analogous result in the nonsink context, represented by the left-most vertical arrow in Figure 1. This arrow represents the fact that the Pak-Stanley labels for the regions of the $G$-semiorder arrangement form the set of quasi-superstables, which is proved in Theorem 23. This theorem is proved by establishing the relations represented by the other arrows in Figure 1.

In working on the G-Shi conjecture of Duval, Klivans, and Martin, we were led to labeling the regions of the $G$-Shi arrangement with partial orientations of $G$. We used Farkas' lemma to develop a rule determining which partial orientations would appear as labels. However, it seemed that the criterion we developed was awkward, and that it would become much simpler and symmetric if we altered the hyperplane arrangement somewhat. This is how we were led to consider $G$-semiorder arrangements. The resulting correspondence between the regions of the $G$-semiorder arrangement and their labels with partial orientations (the $G$-semiorientations) is represented by the top horizontal arrow in Figure 1 and is established in Theorem 14.

The correspondence between maximal superstable configurations - those containing the most amount of sand - and acyclic orientations of the edges of $G$ has been noted several 
times, in different forms ([3], [4], [8], [9]). In [3], the correspondence is a consequence of an extended version of Dhar's burning algorithm, a tool from the chip-firing literature, [6]. The input to their extended algorithm is a maximal superstable configuration, $c$. The configuration is transformed into an unstable configuration $\tilde{c}$. The sequence of vertex firings that stabilizes $\tilde{c}$ is then used to orient the edges of $G$. We modify this algorithm in Section 3.3 to apply to all superstable configurations, not just the maximal ones. This algorithm is encoded (in its nonsink version) as the bottom horizontal and right vertical arrows in Figure 1.

Ultimately, we label each region of the $G$-semiorder arrangement with (i) a $G$-semiorientation, (ii) semiorders on the vertices of $G$, and (iii) a quasi-superstable divisor on $G$. Our aim - to show that all quasi-superstables appear as labels - is achieved by showing that the upper-left and the lower-right triangles in Figure 1 commute and that the mapping referred to as "indeg - 1" along the diagonal in Figure 1 is surjective, (cf. Theorem 23, Corollary 20, and Theorem 19, respectively).

Organization. Following this introduction, the paper is organized into four main sections and a conclusion. Section 1 is an extended example that illustrates our main results and may serve as a foundation for understanding them. The rest of the paper may be considered a justification of the claims made there. Section 2 defines our four main graphical structures. Section 3 contains the central results describing the correspondences between these structures, as discussed above. Section 4 explains how to transfer the results of Section 3 to the context in which a sink vertex is chosen. The concluding section presents a conjecture and suggests further lines of inquiry. Imagine starting with the $G$-semiorder arrangement but then perpendicularly displacing the hyperplanes (replacing the original hyperplanes with parallel ones) before applying the labeling method of Pak and Stanley. As one slides the hyperplanes, some regions disappear and new regions form. We conjecture that as long as a "central region" is preserved, the resulting set of labels does not change. A special case implies the $G$-Shi conjecture of Duval, Klivans, and Martin.

Acknowledgments. We thank Art Duval, Caroline Klivans, and Jeremy Martin for encouraging us to work on the $G$-Shi conjecture and for helpful comments. We thank Collin Perkinson for help with proofreading. We also especially thank the anonymous referee, who helped to improve the quality of our exposition.

\section{Introductory example}

We introduce our main results with an example, beginning with an explanation of the construction of Figure 2. Consider the arrangement of six planes in $\mathbb{R}^{3}$,

$$
x_{i}-x_{j}=1, \quad i, j \in\{1,2,3\}, i \neq j .
$$

The complement of these planes in $\mathbb{R}^{3}$ consists of 19 connected components. The planes are all parallel to the vector $(1,1,1)$, so for our purposes it suffices to intersect the arrangement 


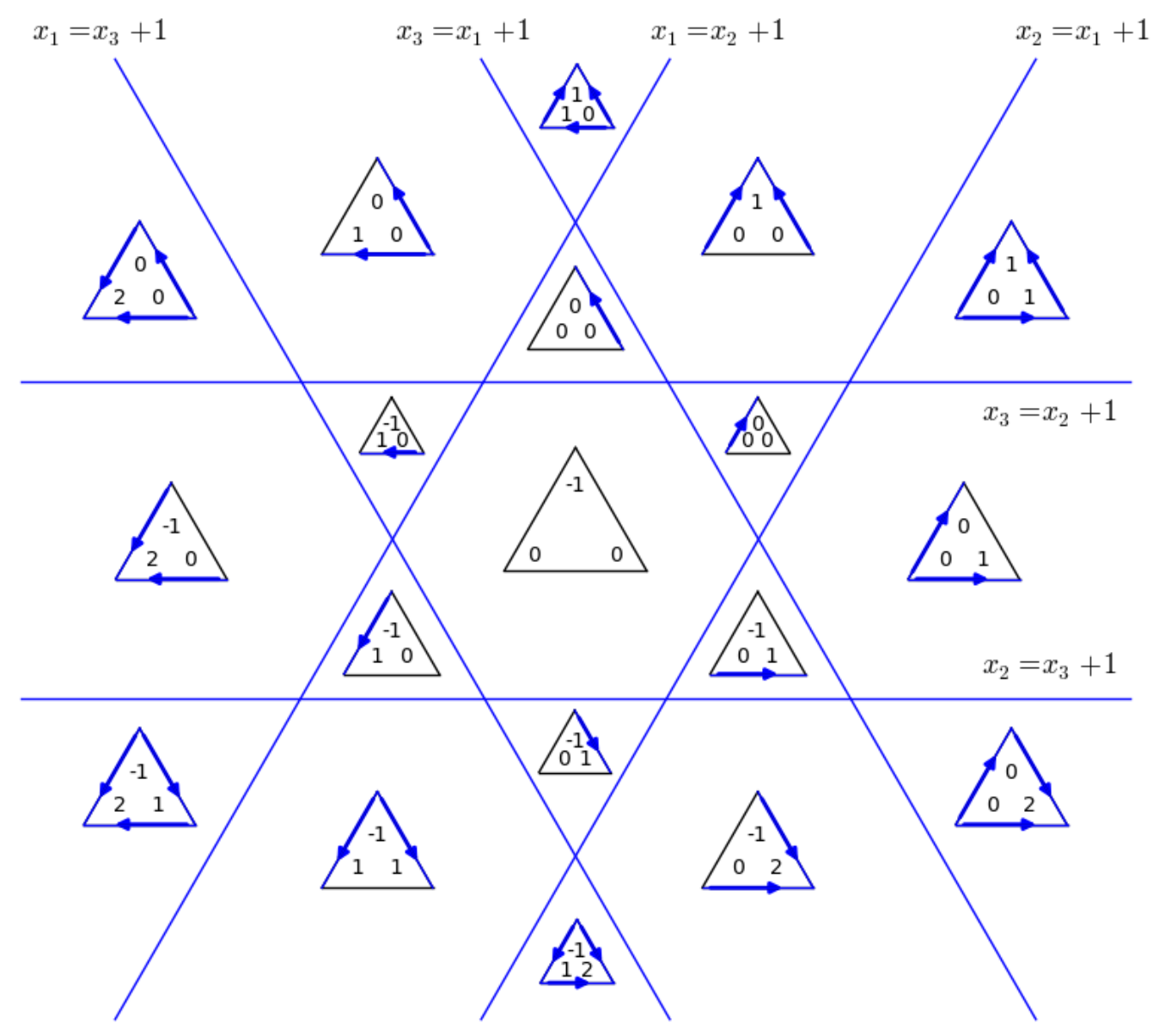

Figure 2: Labeled $(G, q)$-semiorder arrangement.

with the perpendicular plane, $x_{1}+x_{2}+x_{3}=0$, as pictured in Figure 2. Our goal here is to explain the labeling of the 19 regions by vertex-labeled, partially oriented graphs.

To start, each region contains a copy of the graph, $G$, pictured in Figure 3 after removal of the sink vertex, $q$. The vertices of the remaining triangle are labeled by integers, and some of the edges of the triangle are oriented. We refer to the vertex labels as vectors, $\left(c_{1}, c_{2}, c_{3}\right)$, where $c_{i}$ is the label for $v_{i}$, and designate oriented edges as ordered pairs of vertices, $(u, v)$, where $u$ is the tail and $v$ is the head. If there were more room, each region would be labeled by the full graph, $G$. In each region, the label on $q$ would be -1 , and each edge incident on $q$ would be oriented with tail at $q$.

The labeling of the regions is done inductively, starting at the center. In our example, the central region of the arrangement is a hexagon, and its triangle has vertex labels $(0,0,-1)$ and no oriented edges (besides the assumed ones from the sink). The rule for the central region is that a vertex $v_{i}$ has a label of 0 if $\left\{q, v_{i}\right\}$ is an edge; otherwise the label is -1 . More generally, the rule for any region is that the vertex $v_{i}$ is labeled by one less then the number of oriented edges pointing into $v_{i}$. 


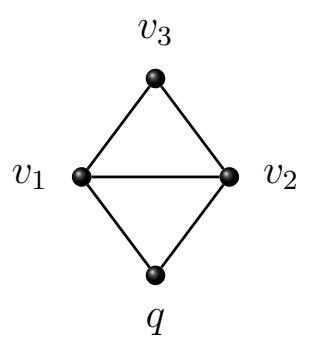

Figure 3: Graph G.

The central region shares an edge with six bordering regions. Let $r$ denote one of these regions, and say that $x_{j}-x_{i}=1$ is the border between it and the central region. Moving from the central region to $r$, one may think of the value of $x_{j}$ as increasing at the expense of $x_{i}$. We record this fact by orienting the edge $\left\{v_{i}, v_{j}\right\}$ as $\left(v_{i}, v_{j}\right)$ and increasing the vertex label for $v_{j}$ by one. Label the five remaining bordering regions similarly. For example, moving into the compact region directly above the central region, we cross into the region where $x_{3}>x_{2}+1$. Thus, the label for this compact region adds an oriented edge $\left(v_{2}, v_{3}\right)$, and the label for $v_{3}$ increases from -1 to 0 .

At this point in the labeling process, there would be six unlabeled regions bordering the six that were just labeled. Let $r$ be one of these unlabeled regions, and let $x_{\ell}-x_{k}=1$ be the edge it shares with a region $r^{\prime}$ that was just labeled. In our case, there are two choices for $r^{\prime}$, but this choice does not affect the eventual label for $r$. To label $r$, start with the label for $r^{\prime}$, add the oriented edge $\left(x_{k}, x_{\ell}\right)$, and increase the vertex label for $v_{\ell}$ by 1 . After labeling these six regions, there are six remaining unlabel regions, and these are labeled by continuing the procedure just described.

In the end, each region is labeled with a copy of $G$ having labeled vertices and a partial orientation of its edges. The resulting partial orientations have a special property. Call an unoriented edge "blank." Consider a collection of edges, $C$, forming a cycle in $G$. Under any partial orientation, some of the edges in $C$ will have an orientation. If it is possible to orient the remaining blank edges in $C$ to get a directed cycle, then the special property is that there must be a greater number of blank edges in $C$ than oriented edges: "more blanks than arrows for potential directed cycles." For example, consider the label for the region just above the central region. It has one oriented edge, $\left(v_{2}, v_{3}\right)$. By orienting the two blank edges as $\left(v_{1}, v_{2}\right)$ and $\left(v_{3}, v_{1}\right)$, we would get a directed cycle, but this potential cycle has two blanks and only one arrow. Theorem 14 guarantees that the 19 regions are in bijection with partial orientations of $G$ having this special property.

As mentioned above, the vertex labels are given as one less than the indegree at each vertex. In our example, there are eight distinct vertex labels with nonnegative entries:

$$
(0,0,0),(1,0,0),(0,1,0),(0,0,1),(2,0,0),(1,0,1),(0,2,0),(0,1,1) .
$$

The surjectivity of the map $\psi$ in Theorem 19 ultimately implies, through Theorem 32, that appending -1 for the sink, $q$, to each of these labels yields the set of $G$-parking functions (with respect to $q$ ). As they are, these labels are exactly the superstable configurations for 
the abelian sandpile model on $G$. Subtracting each from the maximal stable configuration, $(2,2,1)$, gives the recurrent configurations, i.e., the elements of the sandpile group:

$$
(2,2,1),(1,2,1),(2,1,1),(2,2,0),(0,2,1),(1,2,0),(2,0,1),(2,1,0) .
$$

Now pick any region $r$ and a point $t=\left(t_{1}, t_{2}, t_{3}\right) \in r$. The point determines a collection of closed intervals, $I_{i}=\left[t_{i}, t_{i}+1\right]$. Turn the set of intervals into a poset, $P$, by saying $I_{i}<I_{j}$ if $I_{i}$ lies completely to the left of $I_{j}$ with no overlap, i.e., if $t_{i}+1<t_{j}$. Overlapping intervals are not comparable in $P$. Posets arising from finite sets of intervals, ordered in this way, are called semiorders. Identifying interval $I_{i}$ with vertex $v_{i}$ gives a poset on the nonsink vertices, and we then set $q$ to be the unique minimal element to get a poset on all of the vertices.

For instance, the point $p=(1,3,2.5)$ is in the unbounded region directly to the right of the central region in Figure 2, giving the collection of intervals:

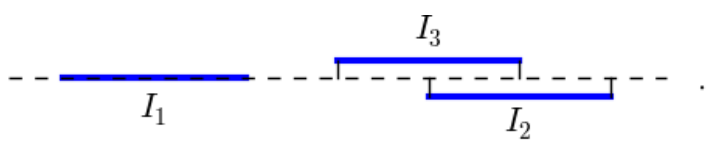

The Hasse diagram for the resulting semiorder on the vertices of $G$ is shown in Figure 4 .

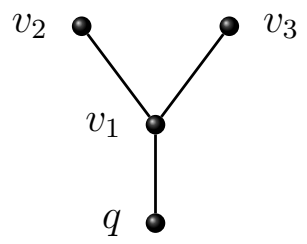

Figure 4: Semiorder determined by $(1,3,2.5) \in \mathbb{R}^{3}$.

The partial orientation of the label for region $r$ can then be read from the semiorder: $\left(v_{i}, v_{j}\right)$ appears as an oriented edge if and only if $\left\{v_{i}, v_{j}\right\}$ is an edge of $G$ and $v_{i}<v_{j}$ in $P$. In general, varying the point $p$ selected in $r$ may result in different semiorders: they may disagree for pairs of vertices that do not determine an edge of $G$. (In our example, each pair of the $v_{i}$ form an edge, so only one poset on the vertices arises from each region.)

How does this example generalize to an arbitrary graph, $G$ ? Suppose $G$ has designated $\operatorname{sink} q$ and has nonsink vertices $v_{1}, \ldots, v_{n}$. Form the arrangement of hyperplanes, $x_{i}-x_{j}=$ 1 for all $i \neq j$ such that $\left\{v_{i}, v_{j}\right\}$ is an edge of $G$. Thus, for each edge, $\left\{v_{i}, v_{j}\right\}$, there is a "stripe" consisting of the two hyperplanes $x_{i}-x_{j}= \pm 1$. The partial orientations in our labeling record on which side of each stripe a given region lies.

Label the central region, for which $\left|x_{i}-x_{j}\right|<1$ for all edges $\left\{v_{i}, v_{j}\right\}$, by the graph $G$, orienting the edges incident with $q$ so that they point away from $q$. Label the $v_{i}$ by the number of oriented edges pointing into $v_{i}$ minus 1 (hence, by 0 or -1 ), and label $q$ with -1 . Then proceed inductively to label all of the regions, as in the example. In the end, the regions will be in bijection with those partial orientations satisfying the property that for each potential directed cycle, there are more blanks than arrows. The collection of vertex 


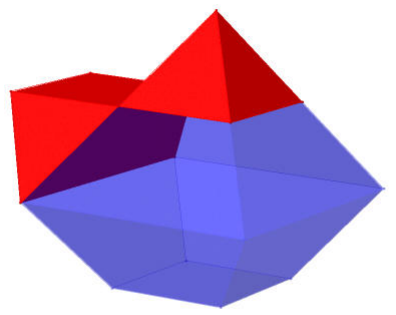

Figure 5: The central region and two bordering regions for $G$.

labels with nonnegative values at each $v_{i}$ are the $G$-parking functions, which correspond to the elements of the sandpile group. Picking a point in any region determines a semiorder on the vertices of $G$, from which one may reconstruct the labeling of $G$ for the region.

We now describe a version of the above construction that avoids an initial choice of a sink. Let $G$ be a graph with vertices $\left\{v_{0}, \ldots, v_{n}\right\}$. Form the hyperplane arrangement as described above: $x_{i}-x_{j}=1$ for each $i \neq j$ such that $\left\{v_{i}, v_{j}\right\}$ is an edge of $G$. Thus, there are two hyperplanes for each edge. Label the central region-given by $\left|x_{i}-x_{j}\right|<1$ for all $i, j$ such that $\{i, j\}$ is an edge of $G$-with a copy of $G$ having no oriented edges and with a -1 at each vertex. Proceed as before, labeling each region. By Theorem 26, those regions for which $v_{i}$ has label -1 , and all other vertices have nonnegative labels, are the $G$-parking functions with respect to $v_{i}$.

For example, again take $G$ to be the graph in Figure 3 but with no vertex chosen as sink (take $q=v_{0}$ ). The corresponding hyperplane arrangement consists of ten hyperplanes in $\mathbb{R}^{4}$. Each hyperplane is parallel to the vector $(1,1,1,1)$, so we project onto the hyperplane given by $\sum_{i=0}^{3} x_{i}=0$, which we identify with $\mathbb{R}^{3}$, to get an arrangement whose 109 regions are in bijection with those of the original arrangement. The central region is a polytope with ten faces. It is pictured in Figure 5 along with two of its bordering regions. The bordering region forming a pyramid on top of the central region is labeled by a copy of $G$ with one directed edge, $\left(v_{2}, v_{1}\right)$, and with vertex label $(-1,0,-1,-1)$. The other bordering region in the figure is labeled by a copy of $G$ with one directed edge, $\left(v_{3}, v_{1}\right)$, and with the same vertex label, $(-1,0,-1,-1)$. Figure 6 depicts those (unbounded) regions of the hyperplane arrangement for $G$ that satisfy $x_{i}>x_{0}+1$ for $i=1,2,3$. They are in bijection with the regions in Figure 2, and corresponding regions would have the same labels. (Recall that for convenience the drawing of the sink vertex and its edges is suppressed in Figure 2.)

\section{Four structures associated with $G$}

From now on, we take $G$ to be a finite, connected, undirected graph, with vertices $V=\left\{v_{0}, \ldots, v_{n}\right\}$ and edges $E$. Loops and multiple edges are disallowed (the former for convenience of notation). 


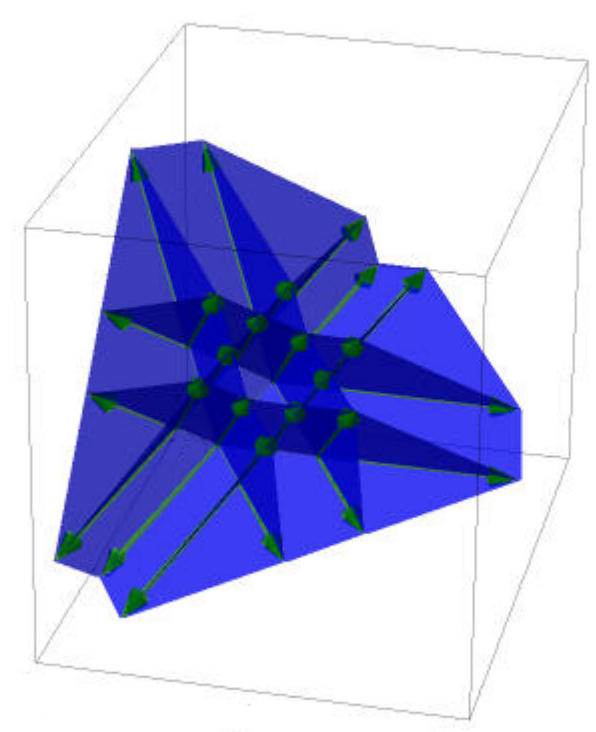

Figure 6: Regions for $G$ corresponding to choosing a sink vertex.

\section{$2.1 \quad G$-semiorientations.}

In this section we define $\mathbb{O}$, the collection of $G$-semiorientations. A partial orientation of $G$ is a choice of directions for a subset of the edges of $G$. Formally, a partial orientation is a subset $\mathcal{O} \subset V \times V$ with the property that if $(u, v) \in \mathcal{O}$, then $\{u, v\} \in E$ and $(v, u) \notin \mathcal{O}$. Let $\mathcal{O}$ be a partial orientation. If $e=\{u, v\} \in E$ and $(u, v) \in \mathcal{O}$, then despite the ambiguity, we write $e \in \mathcal{O}$ and say $e$ is oriented. In that case, we think of $e$ as an arrow from $u$ to $v$ and write $e^{-}=u$ and $e^{+}=v$. If neither $(u, v)$ nor $(v, u)$ is in $\mathcal{O}$, we write $e \notin \mathcal{O}$ and say that $e$ is an unoriented or blank edge. The outdegree of the vertex $u \in V$ relative to $\mathcal{O}$, denoted $\operatorname{outdeg}_{\mathcal{O}}(u)$, is the number of edges $e \in \mathcal{O}$ such that $e^{-}=u$.

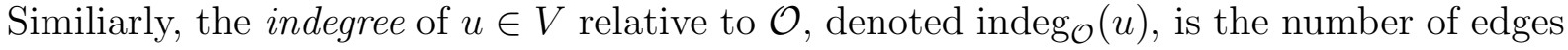
$e \in \mathcal{O}$ such that $e^{+}=u$. We use the notation $\operatorname{deg}(u)$ to denote the ordinary degree of $u$, i.e., the number of $e \in E$ containing $u$. Fixing $\mathcal{O}$, some of the edges of any cycle $C \subseteq E$ of $G$ will be oriented and others will be blank. If it is possible to assign directions to the blank edges so that $C$ would become a directed cycle, then we call $C$ a potential cycle for $\mathcal{O}$.

Definition 1. A $G$-semiorientation is a partial orientation, $\mathcal{O}$, such that each potential cycle for $\mathcal{O}$ has more blank edges than oriented edges. The set of $G$-semiorientations of $G$ is denoted $\mathbb{O}$.

\section{$2.2 \quad G$-semiorders.}

A reference for ordinary semiorders is [17]. Let $k$ be any nonnegative integer, and consider a collection of unit length closed intervals, $P=\left\{I_{1}, \ldots, I_{k}\right\}$, of the real line. Order the elements of $P$ by $I_{i}<I_{j}$ if $I_{i}$ lies strictly to the left of $I_{j}$, i.e., if $I_{i}=\left[a_{i}, a_{i}+1\right]$ and $I_{j}=\left[a_{j}, a_{j}+1\right]$, then $a_{i}+1<a_{j}$. Any poset isomorphic to a poset $P$, constructed as 
above, is called a semiorder. The number of non-isomorphic semiorders with $k$ elements is the $k$-th Catalan number, $C_{k}$, and there is a corresponding generating function

$$
C(x)=\sum_{k \geqslant 0} C_{n} x^{k}=\frac{1-\sqrt{1-4 x}}{2 x} .
$$

If $f_{k}$ denotes the number of labeled semiorders, there is the exponential generating function

$$
\sum_{k \geqslant 0} f_{k} \frac{x^{k}}{k !}=C\left(1-e^{-x}\right) .
$$

Definition 2. A $G$-semiorder is a semiorder on the vertices of $G$. The set of $G$-semiorders is denoted $\mathbb{I}$.

\subsection{The $G$-semiorder arrangement.}

Definition 3. The $G$-semiorder arrangement, denoted $\mathscr{I}$, is the set of $2|E|$ hyperplanes in $\mathbb{R}^{n+1}$ given by

$$
x_{i}-x_{j}=1,
$$

for all $i \neq j$ such that $\left\{v_{i}, v_{j}\right\} \in E$. The regions of $\mathscr{I}$, denoted $\mathcal{R}$, are the connected components of $\mathbb{R}^{n+1} \backslash \mathscr{I}$.

If $G$ were the complete graph on $n+1$ vertices, the $G$-semiorder arrangement would be the ordinary semiorder arrangement discussed in [17], whose regions are in bijection with labeled semiorders on $n+1$ elements. For general $G$, each region of the $G$-semiorder arrangement is a union of regions from the ordinary semiorder arrangement.

\subsection{Quasi-superstables on $G$.}

Now designate $q:=v_{0}$ as the sink vertex. We recall the basic facts about the abelian sandpile model on $G$, including $G$-parking functions. A reference for the sandpile results stated here is [10]. (More references for the abelian sandpile model: [2] and [5] are seminal; [6] and [13] are general references; and [12] is a quick overview.) We then proceed to define $\mathcal{S}$, the set of quasi-superstable divisors of $G$.

Let $D=\operatorname{diag}\left(\operatorname{deg} v_{0}, \ldots, \operatorname{deg} v_{n}\right)$, and let $A$ be the adjacency matrix for $G$, defined by

$$
A_{i j}= \begin{cases}1 & \text { if }\left\{v_{i}, v_{j}\right\} \in E \\ 0 & \text { otherwise }\end{cases}
$$

The Laplacian matrix for $G$ is

$$
\Delta=D-A .
$$

The reduced Laplacian is the matrix $\widetilde{\Delta}$ obtained by removing the first row and column of $\Delta$, i.e., the row and column corresponding to the sink vertex. 
A configuration on $G$ is an element of the free abelian group on the nonsink vertices. Having ordered the nonsink vertices, as above, we identify the set of configurations with $\mathbb{Z}^{n}$ in the natural way: $c=\sum_{i=1}^{n} c_{i} v_{i} \leftrightarrow\left(c_{1}, \ldots, c_{n}\right)$. A divisor on $G$ is an element of the free abelian group on all of the vertices of $G$, which we similarly identify with $\mathbb{Z}^{n+1}$. Once a sink is chosen, we may consider configurations as those divisors whose sink coefficient is 0 .

Given two configurations or two divisors $c$ and $c^{\prime}$, we write $c \geqslant c^{\prime}$ if $c_{i} \geqslant c_{i}^{\prime}$ for all $i$. We say $c$ is nonnegative if $c \geqslant 0$, i.e., if each component of $c$ is nonnegative.

If $X$ is a subset of the nonsink vertices, write $1_{X}$ for the configuration whose $i$-th component is 1 if $v_{i} \in X$ and 0 otherwise. Firing $X$ from a configuration $c$ results in the configuration $c-\widetilde{\Delta} 1_{X}$. If $X=\left\{v_{i}\right\}$, we call this operation firing $v_{i}$. One may speak of firing the sink vertex, which adds 1 to each vertex connected to the sink. Since the sum of the columns of the Laplacian matrix is zero, firing the set of all nonsink vertices is the same as reverse-firing the sink, subtracting 1 from each vertex attached to the sink.

Definition 4. Let $c$ be a nonnegative configuration on $G$. We say $c$ is stable if there is no $i$ such that firing $v_{i}$ from $c$ results in a nonnegative configuration. We say that $c$ is superstable if there is no nonempty subset $X$ of the nonsink vertices such that firing $X$ from $c$ results in a nonnegative configuration.

The notion of a superstable configuration is essentially the same as that of a parking function.

Definition 5. A function $f: V \rightarrow \mathbb{Z}$ is a $G$-parking function (with respect to $q$ ) if $f(q)=$ -1 and $\left(f\left(v_{1}\right), \ldots, f\left(v_{n}\right)\right)$ is a superstable configuration on $G$. We identify a $G$-parking function $f$ with the divisor $\sum_{v \in V} f(v) v$.

Suppose $c$ is a nonnegative configuration. Then $c$ is stable exactly when $c_{i}<\operatorname{deg} v_{i}$ for all $i$. A vertex $v_{i}$ such that $c_{i}<\operatorname{deg} v_{i}$ is said to be stable in $c$; otherwise it is unstable. Firing a set of vertices is legal from $c$ if the resulting configuration is nonnegative. In particular, firing a single unstable vertex of $c$ is legal. A sequence of vertices is called a legal firing sequence for $c$ if each vertex in the sequence is unstable after firing the previous vertices in the sequence. Since there is a path from each nonsink vertex to the sink in $G$, there is a legal firing sequence leading to a stable configuration $c^{\circ}$ called the stabilization of $c$. This process is called stabilizing $c$. It turns out that $c^{\circ}$ is independent of the order in which unstable vertices are fired, as is the number of times each vertex is fired in reaching $c^{\circ}$.

Definition 6. A stable configuration $c \geqslant 0$ is recurrent if given any nonnegative configuration $a$, there exists a nonnegative configuration $b$ such that $c=(a+b)^{\circ}$. The recurrent elements with the operation of (vertex-wise) addition followed by stabilization is called the sandpile group of $G$ (with respect to $q$ ), denoted $\operatorname{Sand}(G)$.

It is well-known that the sandpile group actually is a group and the mapping

$$
\begin{aligned}
\operatorname{Sand}(G) & \rightarrow \mathbb{Z}^{n} / \operatorname{image}(\widetilde{\Delta}) \\
c & \mapsto c
\end{aligned}
$$


is an isomorphism. Each equivalence class of $\mathbb{Z}^{n}$ modulo the image of the reduced Laplacian contains a unique recurrent element. It is also known that each equivalence class contains a unique superstable element. Define the maximal stable configuration to be

$$
c_{\max }=\sum_{i=1}^{n}\left(\operatorname{deg} v_{i}-1\right) v_{i} .
$$

The next two propositions are well-known.

Proposition 7 ([10, Theorem 4.4]). The configuration $c$ is recurrent if and only if $c_{\max }-c$ is superstable.

Proposition 8 (Dhar's burning algorithm, [6], [10, Lemma 4.1]). Let $b \geqslant 0$ be a stable configuration on $G$, and let $\tilde{b}$ be the configuration obtained from $b$ by firing the sink. The following are equivalent:

1. $b$ is recurrent,

2. $(\tilde{b})^{\circ}=b$, i.e., the stabilization of $\tilde{b}$ is $b$,

3. in stabilizing $\tilde{b}$, each nonsink vertex fires exactly once.

Remark. We refer to Proposition 8 as Dhar's burning algorithm, although it would more properly be called the theoretical underpinning of the algorithm.

Definition 9. Let $K(G)$ be the graph $G$ with the addition of a new vertex $\tilde{q}$ and edges $\left\{\tilde{q}, v_{i}\right\}$ for all vertices $v_{i}$, including $q=v_{0}$. Set $\tilde{q}$ as the sink vertex of $K(G)$.

Thus, the set of nonsink vertices of $K(G)$ is $V$, and each of these is connected to the sink, $\tilde{q}$, by an edge. The divisors on $G$ are exactly the configurations on $K(G)$.

Definition 10. A divisor $c \in \mathbb{Z}^{n+1}$ on $G$ is called quasi-superstable if $c=\tilde{c}-1_{V}$ for some superstable $\tilde{c}$ on $K(G)$. The collection of quasi-superstable divisors is denoted $\mathcal{S}$.

For the relation between the superstables and quasi-superstables of $G$, see Theorem 26 (2).

\section{Correspondences between the structures}

So far, we have defined the following structures on $G$ :

$$
\begin{aligned}
\mathbb{O}: & G \text {-semiorientations, } \\
\mathbb{I}: & G \text {-semiorders, } \\
\mathscr{I}: & \text { the } G \text {-semiorder arrangement, } \\
\mathcal{S}: & \text { quasi-superstable divisors on } G .
\end{aligned}
$$

In this section, we describe relations among these structures, culminating in Theorems 19 and 23 . 


\subsection{Semiorders and semiorientations}

Definition 11. A $G$-semiorder $P$ and a $G$-semiorientation $\mathcal{O}$ are compatible if for each edge $e=\{u, v\}$ of $G$, we have that $u<v$ if and only if $(u, v) \in \mathcal{O}$. Thus, if $e \notin \mathcal{O}$, then $u$ and $v$ are not comparable in $P$.

Given a $G$-semiorder, $P$, define

$$
\mathcal{O}_{P}=\{(u, v):\{u, v\} \in E \text { and } u<v \text { in } P\} .
$$

Theorem 12. Let $P$ be a G-semiorder. Then $\mathcal{O}_{P} \in \mathbb{O}$ and $\mathcal{O}_{P}$ is the unique element of (O) compatible with $P$.

Proof. The only part of this theorem that is not immediate from the definitions is the fact that every potential cycle for $\mathcal{O}_{P}$ has more blanks edges than oriented edges, which we now prove. Let $\alpha=\left\{e_{1}, \ldots, e_{k}\right\}$ be a potential cycle. We may assume that $e_{i}=\left\{u_{i}, u_{i+1}\right\}$ where $u_{k+1}=u_{1}$, and that for each $i$, either (i) $\left(u_{i}, u_{i+1}\right) \in \mathcal{O}_{P}$, in which case $u_{i}<u_{i+1}$, or (ii) $e_{i}$ is a blank edge, in which case $u_{i}$ and $u_{i+1}$ are not comparable. Since $P$ is a semiorder, it is isomorphic to a semiorder of intervals, allowing us to identify each $u_{i}$ with an interval $I_{i}=\left[a_{i}, a_{i}+1\right]$. If $e_{i} \in \mathcal{O}_{P}$, we have $I_{i}<I_{i+1}$, in which case $a_{i}<a_{i+1}-1$; and if $e_{i} \notin \mathcal{O}_{P}$, then $I_{i}$ and $I_{i+1}$ overlap, so in particular, $a_{i} \leqslant a_{i+1}+1$. Thus, if there are $\delta$ oriented edges and $\beta$ blank edges in $\alpha$, since $u_{k+1}=u_{1}$,

$$
a_{1}+\delta-\beta \leqslant a_{1}
$$

with equality if and only if $\beta=\delta=0$. However, since $\alpha$ has at least one edge, the inequality must be strict, and $\beta>\delta$ as required.

Thus, we can refer to the semiorientation determined by a $G$-semiorder and define the mapping

$$
\begin{aligned}
\nu: \mathbb{I} & \rightarrow \mathbb{O} \\
P & \mapsto \mathcal{O}_{P} .
\end{aligned}
$$

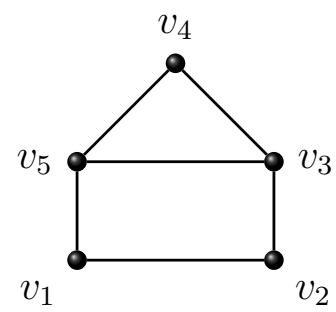

Figure 7: The house graph.

Example 13. Let $G$ be the house graph of Figure 7. Figure 8 depicts a $G$-semiorder and its corresponding compatible $G$-semiorientation. 


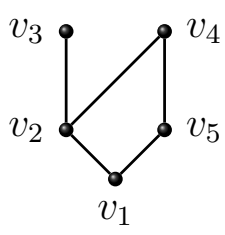

$P$

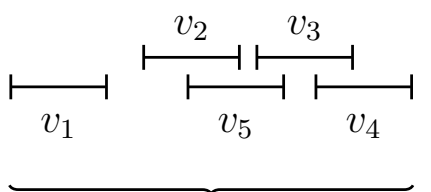

unit intervals

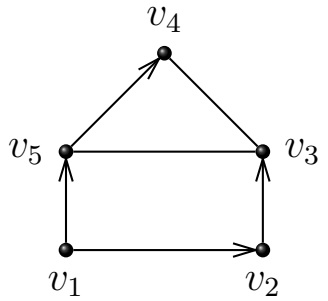

$\mathcal{O}_{P}$

Figure 8: A $G$-semiorder $P$ on the vertices of the house graph, $G$; a collection of intervals realizing the semiorder; and the unique $G$-semiorientation, $\nu(P)=\mathcal{O}_{P}$, compatible with $P$.

\subsection{A bijection between semiorientations and hyperplane re- gions}

We now define a mapping

$$
\rho: \mathbb{O} \rightarrow \mathcal{R} \text {. }
$$

If $\mathcal{O} \in \mathbb{O}$, let $\rho(\mathcal{O})$ be the region defined by the following inequalities: for each edge $e$ of $G$ :

- if $e=\left(v_{i}, v_{j}\right) \in \mathcal{O}$, then $x_{j}>x_{i}+1$,

- if $e \notin \mathcal{O}$, then $\left|x_{i}-x_{j}\right|<1$.

The reader may find it helpful to skim the example below the proof of the following theorem before reading the proof itself.

Theorem 14. The mapping $\rho$ is a well-defined bijection.

Proof. Let $\mathcal{O} \in \mathbb{O}$, and let $r=\rho(\mathcal{O})$. The system of inequalities defining $r$ indicates on which side of each hyperplane of $\mathscr{I}$ the region sits. To show $r$ is a region of $\mathscr{I}$, it suffices to show that $r$ is nonempty.

We define a directed, weighted graph $G^{\prime}$ with the same vertices as $G$ in the following manner: for each edge $e$ between vertices of $G$,

- if $e=\left(v_{i}, v_{j}\right) \in \mathcal{O}$, then $\left(v_{i}, v_{j}\right) \in G^{\prime}$ and the weight of $\left(v_{i}, v_{j}\right)$ in $G^{\prime}$ is -1 ,

- if $e=\left\{v_{i}, v_{j}\right\} \notin \mathcal{O}$, then $\left(v_{i}, v_{j}\right),\left(v_{j}, v_{i}\right) \in G^{\prime}$ and the weight of both $\left(v_{i}, v_{j}\right)$ and $\left(v_{j}, v_{i}\right)$ in $G^{\prime}$ is 1 .

Choose an ordering of the edges of $G^{\prime}: e_{1}^{\prime}, e_{2}^{\prime}, \ldots, e_{k}^{\prime}$. Define a $k \times(n+1)$ edge-vertex adjacency matrix with rows $r_{1}, \ldots, r_{k}$ as follows: if $e_{\ell}^{\prime}=\left(v_{i}, v_{j}\right)$, let $r_{\ell}$ be the vector having 1 in the $i$ th entry, -1 in the $j$ th entry, and 0 s elsewhere. Let $b$ be a column vector in $\mathbb{R}^{k}$ where $b_{\ell}$ is the weight of $e_{\ell}^{\prime}$. 
Thus, the inequalities of $\rho(\mathcal{O})$ are encoded as $A x<b$. By Farkas' lemma the insolvability of $A x<b$ is equivalent to the existence of a row vector $y=\left(y_{1}, \ldots, y_{k}\right)$ satisfying:

$$
y_{i} \geqslant 0 \quad \forall i, \quad y \neq 0, \quad y A=0, \quad y \cdot b \leqslant 0 .
$$

For sake of contradiction, suppose such a $y$ exists. The support of $y$ is

$$
\operatorname{supp}(y)=\left\{i: y_{i} \neq 0\right\} .
$$

Among all row vectors satisfying condition (1), suppose $y$ has been chosen so that the cardinality of its support is minimal. Say $\ell_{1} \in \operatorname{supp}(y)$ and $e_{\ell_{1}}^{\prime}=\left(v_{i}, v_{j}\right)$. Hence, $r_{\ell_{1}}$ has a -1 in its $v_{j}$-th entry. Since

$$
y A=y_{1} r_{1} \cdots+y_{k} r_{k}=0
$$

and the components of $y$ are nonnegative, there must be some $\ell_{2} \in \operatorname{supp}(y)$ such that $r_{\ell_{2}}$ has a 1 in its $v_{j}$-th entry. This row will have a -1 in some other entry, forcing the existence of some $\ell_{3} \in \operatorname{supp}(y)$ such that $r_{\ell_{3}}$ has a 1 in that entry, and so on. Since the support of $y$ is finite, the sequence $\ell_{1}, \ell_{2}, \ldots$, eventually has a repeat. Thus, there is a sequence of elements $j_{1}:=\ell_{m+1}, j_{2}:=\ell_{m+2}, \ldots, j_{t}:=\ell_{m+t}$ in the support of $y$ for some $t$ corresponding to a directed cycle of edges $e_{j_{1}}^{\prime}, \ldots, e_{j_{t}}^{\prime}$ in $G^{\prime}$.

Let $z=\left(z_{1}, \ldots, z_{k}\right)$ be the row vector with $z_{\ell}=1$ if $\ell \in\left\{j_{1}, \ldots, j_{t}\right\}$ and $z_{\ell}=0$, otherwise. Since the support of $z$ corresponds to a directed cycle of edges in $G^{\prime}$, we have $z A=0$. Furthermore, since any potential cycle in $\rho(\mathcal{O})$ has more blank edges than oriented edges, we have $z \cdot b>0$. Let $a=\min \left\{y_{j_{1}}, \ldots, y_{j_{t}}\right\}$ and define $y^{\prime}=y-a z$. Then $y^{\prime}$ satisfies condition (1) but its support is strictly contained in the support of $y$, yielding a contradiction. So there must be some solution to $A x<b$, which means that $r=\rho(\mathcal{O}) \in \mathcal{R}$.

We now define a mapping

$$
\tau: \mathcal{R} \rightarrow \mathbb{O} \text {. }
$$

If $r \in \mathcal{R}$ let $\tau(r)$ be the partial orientation of $G$ obtained by (i) $\left(v_{i}, v_{j}\right) \in \tau(r)$ if $\left\{v_{i}, v_{j}\right\} \in E$ and $x_{j}>x_{i}+1$ in $r$, and (ii) all other edges of $G$ are blank. Once we show $\tau$ is well-defined, it is immediate that it is the inverse of $\rho$.

Let $r \in \mathcal{R}$. To see that $\tau(r) \in \mathbb{O}$, suppose $\tau(r)$ has a potential cycle $\alpha$ having at least as many oriented edges as blank edges. Define $A$ and $b$ as above to encode the system of inequalities that defines the region $r$ as $A x<b$. Let $y$ be the row vector with 1 s in the entries corresponding to the (oriented and blank) edges of $\alpha$ and 0s elsewhere. We have $y \geqslant 0, y \neq 0, y A=0$, and $y \cdot b \leqslant 0$. But by Farkas' lemma this means $A x<b$ has no solutions, contradicting the fact that $r \in \mathcal{R}$. Thus, $\tau(r) \in \mathbb{O}$.

Example 15. This example is intended to be read in conjunction with the proof of Theorem 14 and illustrates Farkas' lemma at work. Consider the partial orientation, $\mathcal{O}$, of a triangle, pictured in Figure 9. Naively attemtping to apply the region-labeling function, $\rho$, 


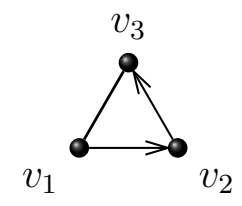

Figure 9: Partial orientation, $\mathcal{O}$.

to $\mathcal{O}$ results in the system of inequalities $A x<b$ where

$$
A=\left[\begin{array}{rrr}
1 & -1 & 0 \\
0 & 1 & -1 \\
1 & 0 & -1 \\
-1 & 0 & 1
\end{array}\right], \quad x=\left[\begin{array}{l}
x_{1} \\
x_{2} \\
x_{3}
\end{array}\right], \quad b=\left[\begin{array}{r}
-1 \\
-1 \\
1 \\
1
\end{array}\right] .
$$

The rows of $A$ correspond to directed edges, $\left(v_{1}, v_{2}\right),\left(v_{2}, v_{3}\right),\left(v_{1}, v_{3}\right)$, and $\left(v_{3}, v_{1}\right)$, respectively, of the underlying triangle, $G$. The last two inequalities combine to describe the "sandwich" $\left|x_{1}-x_{3}\right|<1$, corresponding to the undirected edge, $\left\{v_{1}, v_{3}\right\}$.

The partial orientation, $\mathcal{O}$, has a potential cycle with more oriented edges than blanks, and hence is not a $G$-semiorientation. By the way in which $A$ is defined in the proof of Theorem 14, we know that adding the rows of $A$ corresponding to the directed cycle $\left(v_{1}, v_{2}\right),\left(v_{2}, v_{3}\right),\left(v_{3}, v_{1}\right)$ gives the zero vector. Our system of inequalities is inconsistent since adding the corresponding entries of $b$ gives -1 (since there are more oriented edges than blanks in $\mathcal{O}$ for this cycle).

Let $r$ be a region in the image of $\rho$. If $t=\left(t_{0}, \ldots, t_{n}\right) \in r$, define the unit intervals $I_{i}=\left[t_{i}, t_{i}+1\right]$ for $i=0, \ldots, n$. Define $P_{t}$ to be the $G$-semiorder determined by these intervals, labeled by the vertices of $G$ by identifying $I_{i}$ with $v_{i}$.

Theorem 16. Let $\mathcal{O} \in \mathbb{O}$, and let $r=\rho(\mathcal{O})$. The semiorders $P_{t}$ as $t$ ranges over points in $r$ are exactly the $G$-semiorders compatible with $\mathcal{O}$.

Proof. Choose any $t \in r$. Say $\left(v_{i}, v_{j}\right) \in \mathcal{O}$. Then $x_{j}>x_{i}+1$ in $r$; so $t_{j}>t_{i}+1$, and hence, $v_{i}<v_{j}$ in $P_{t}$. Now suppose $e=\left\{v_{i}, v_{j}\right\} \in E$ but $e \notin \mathcal{O}$. Then $\left|t_{i}-t_{j}\right|<1$, which means that $I_{i}$ and $I_{j}$ overlap, and hence, $v_{i}$ and $v_{j}$ are not comparable in $P_{t}$. This shows that $P_{t}$ is compatible with $\mathcal{O}$.

Now let $P$ be a $G$-semiorder compatible with $\mathcal{O}$. The semiorder $P$ is isomorphic to the semiorder on a set of unit intervals, $\left\{I_{i}\right\}_{i=0}^{n}$, where $I_{i}$ corresponds to $v_{i}$. Say $I_{i}=\left[t_{i}, t_{i}+1\right]$ for each $i$, and let $t=\left(t_{0}, \ldots, t_{i}\right)$. So $P=P_{t}$, but we must show that $t \in r$. Suppose $e=\left\{v_{i}, v_{j}\right\} \in E$. If $x_{j}>x_{i}+1$ in $r$, then since $\mathcal{O}=\tau(r)$, we have $\left(v_{i}, v_{j}\right) \in \mathcal{O}$, and thus $t_{j}>t_{i}+1$. If $\left|x_{i}-x_{j}\right|<1$, then $e \notin \mathcal{O}$ and the intervals $I_{i}$ and $I_{j}$ overlap, i.e., $\left|t_{i}-t_{j}\right|<1$. Hence, $t \in r$.

Example 17. Let $G$ be the house graph pictured in Figure 7, and let $\mathcal{O}$ be the $G$ semiorientation pictured on the right in Figure 8. The region $\rho(\mathcal{O})$ corresponding to $\mathcal{O}$ is 


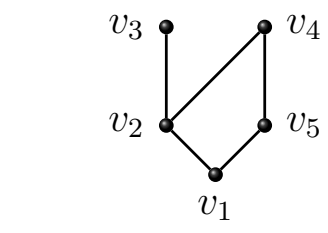

$P_{t}$

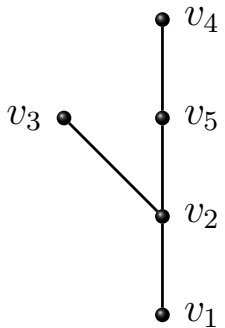

$P_{t^{\prime}}$

$$
t=(0,1.5,2.75,3.5,2) \quad t^{\prime}=(0,2,4.75,5.5,4)
$$

Figure 10: Two semiorders.

determined by the system of inequalities:

$$
\begin{aligned}
& x_{2}>x_{1}+1, \quad x_{3}>x_{2}+1, \\
& x_{4}>x_{5}+1, \quad x_{5}>x_{1}+1, \\
& \left|x_{3}-x_{4}\right|<1, \quad\left|x_{3}-x_{5}\right|<1 .
\end{aligned}
$$

Figure 10 displays points $t, t^{\prime} \in \rho(\mathcal{O})$ whose corresponding semiorders, $P_{t}$ and $P_{t^{\prime}}$, are the two $G$-semiorders compatible with $\mathcal{O}$.

\subsection{Superstables algorithm}

So far, we have described relations among three of our four structures: $G$-semiorders, $G$-semiorientations, and the regions of the $G$-semiorder arrangement. We would now like to forge a connection between these and the quasi-superstables on $G$ (ultimately relating all of this, in Section 4, to ordinary superstables).

Dhar's burning algorithm says that starting from a recurrent configuration, then firing the sink, each non-sink vertex will fire exactly once in the stabilization process. We use these vertex firings to build a partial orientation starting from the unoriented graph. To sketch the idea, suppose that at some point in the stabilization process there are nonsink vertices $v$ and $w$ such that $e=\{v, w\}$ is an edge and $v$ is unstable. Suppose $e$ has not already been oriented or marked as a blank edge. When $v$ fires, if $w$ is stable, orient $e$ as $(v, w)$; otherwise mark $e$ blank. Since all vertices fire, each edge is visited.

Starting with a quasi-superstable, $c$, on $G$, Proposition 7 provides a corresponding recurrent configuration on $K(G)$. We use Dhar's algorithm as above to create a $G$ semiorientation, $\mathcal{O}$, of $G$. Using the construction of $\mathcal{O}$ as a guide, one may further refine the procedure to simultaneously create a $G$-semiorder compatible with $\mathcal{O}$. This is the idea behind the intervals labeled by $J$ in the algorithm, below. Theorem 19 then provides the important connection: subtract one from each entry of the indegree sequence relative to $\mathcal{O}$ to recapture $c$. 
A careful description of our algorithm follows. The input is a quasi-superstable divisor on $G$ and a vertex ordering, and the output is a $G$-semiorder $P$ and the $G$ semiorientation $\mathcal{O}_{P}$ compatible with $P$. Letting $\mathfrak{S}$ be the symmetric group on $\{0, \ldots, n\}$, identify $\sigma \in \mathfrak{S}$ with the vertex ordering $\left(v_{\sigma(0)}, \ldots, v_{\sigma(n)}\right)$. Then our algorithm defines two mappings $\phi: \mathcal{S} \times \mathfrak{S} \rightarrow \mathbb{I}$ and $\eta: \mathcal{S} \times \mathfrak{S} \rightarrow \mathbb{O}$. Theorem 19, to follow, validates the algorithm and shows that it produces a commutative diagram

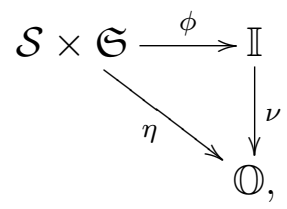

where $\nu$ is the mapping defined in Section 3.1.

Given a $c \in \mathcal{S}$ and a vertex ordering $\sigma \in \mathfrak{S}$, the algorithm proceeds as follows:

\section{INITIALIZATION}

Let $c_{\max }$ be the maximal stable configuration of $K(G)$, and let

$$
b=c_{\max }-c .
$$

Since $c \in \mathcal{S}$, we can write $c=\tilde{c}-1_{V}$ for some superstable $\tilde{c}$ on $K(G)$. Therefore, $b=\left(c_{\max }-\tilde{c}\right)+1_{V}$, i.e., $b$ is the configuration on $K(G)$ obtained from $c_{\max }-\tilde{c}$ by firing the sink $\tilde{q}$ of $K(G)$. By Proposition $7, c_{\max }-\tilde{c}$ is a recurrent configuration on $K(G)$; so by Proposition 8 , every element of $V$ will fire exactly once while stabilizing $b$.

Let $u_{1}, \ldots, u_{k}$ be the vertices that are unstable in $b$. Take this list of vertices to be ordered according to $\sigma$, that is, if $u_{i}=v_{\sigma(\ell)}$ and $u_{j}=v_{\sigma(m)}$ with $\ell<m$, then $i<j$. For each $u_{i}$, associate an interval,

$$
J\left(u_{i}\right)=[i /(k+1), 1+i /(k+1)] .
$$

Thus, all the $J\left(u_{i}\right)$ overlap. Form a queue,

$$
Q=\left(u_{1}, \ldots, u_{k}\right)
$$

Initialize the partial orientation of $G$ as $\mathcal{O}=\emptyset$.

$$
\text { LOOP }
$$

Repeat the following until the queue is empty:

Say the queue is $Q=\left(w_{0}, \ldots, w_{\ell}\right)$, and

$$
J\left(w_{0}\right)=[\alpha, \alpha+1] .
$$

If $\ell>0$, define $\varepsilon$ using the interval for $w_{1}$ :

$$
J\left(w_{1}\right)=[\alpha+\varepsilon, \alpha+\varepsilon+1] .
$$


Otherwise, take $\varepsilon=1$.

Fire $w_{0}$ and replace $b$ by the resulting configuration. Remove $w_{0}$ from the queue and mark it so that it will never again appear in the queue.

Let $z_{1}, \ldots, z_{t}$ be the vertices that just became unstable with the firing of $w_{0}$ and that have not yet been fired by the algorithm, listed in order according to $\sigma$. Define

$$
J\left(z_{i}\right)=[\alpha+1+i \varepsilon /(t+1), \alpha+2+i \varepsilon /(t+1)] .
$$

Add the $z_{i}$ to $Q$, in order:

$$
Q=\left(w_{1}, \ldots, w_{\ell}, z_{1}, \ldots, z_{t}\right)
$$

For each edge $e=\left\{w_{0}, v\right\}$ that is not oriented or marked as blank,

1. if $v$ was already unstable before the firing of $w_{0}$, mark $e$ as blank, so $e$ will not subsequently be added to $\mathcal{O}$;

2. otherwise orient the edge out from $v$, i.e., add $\left(w_{0}, v\right)$ to $\mathcal{O}$.

\section{OUTPUT}

The intervals $\{J(v): v \in V\}$ determine a semiorder. Identifying $v$ with $J(v)$ gives a semiorder, $P$, on $V$. Define $\phi(c)=P$ and $\eta(c)=\mathcal{O}$.

Example 18. Let $G$ be the house graph of Figure 7, and fix the vertex ordering, $v_{1}, \ldots, v_{5}$. Then $c=(-1,0,0,0,0)$ is a quasi-superstable divisor on $G$. Figure 11 illustrates the application of the superstables algorithm to $c$ and the given vertex ordering, producing a $G$-semiorientation, $\mathcal{O}$. The dotted lines emanating from each vertex to the exterior of the house graph represent the edges to the sink of $K(G)$.

We have $c_{\max }=(2,2,3,2,3)$ for $K(G)$. The algorithm starts with the configuration $b=c_{\max }-c$ in the top left corner of Figure 11. The vertex $v_{1}$ is unstable in $b$, and firing produces two oriented edges and two unstable vertices, $v_{2}$ and $v_{5}$. Since $v_{2}$ comes first in the vertex ordering, it is fired next. The subscripts keep track of vertex-firing precedence. Note that when $v_{5}$ is fired, the edge $\left\{v_{3}, v_{5}\right\}$ is marked blank since, by that time, $v_{3}$ is unstable. Proceeding clockwise around the diagram, the algorithm terminates at the bottom left corner.

Let $\tilde{c}:=c+1_{V}$, a superstable configuration on $K(G)$. When the algorithm terminates, the resulting configuration is $c_{\max }-\tilde{c}$, which is recurrent by Proposition 7 . Adding $1_{V}$ reproduces the starting configuration $c$. However, adding $1_{V}$ is equivalent to firing the sink of $K(G)$, which explains (via Dhar's burning algorithm) why every vertex was guaranteed to fire.

Theorem 19. Given $P \in \mathbb{I}$, for each vertex $v$ let $n_{P}(v)$ denote the cardinality of the set

$$
\{u \in V: u<v \text { and }\{u, v\} \in E\} .
$$



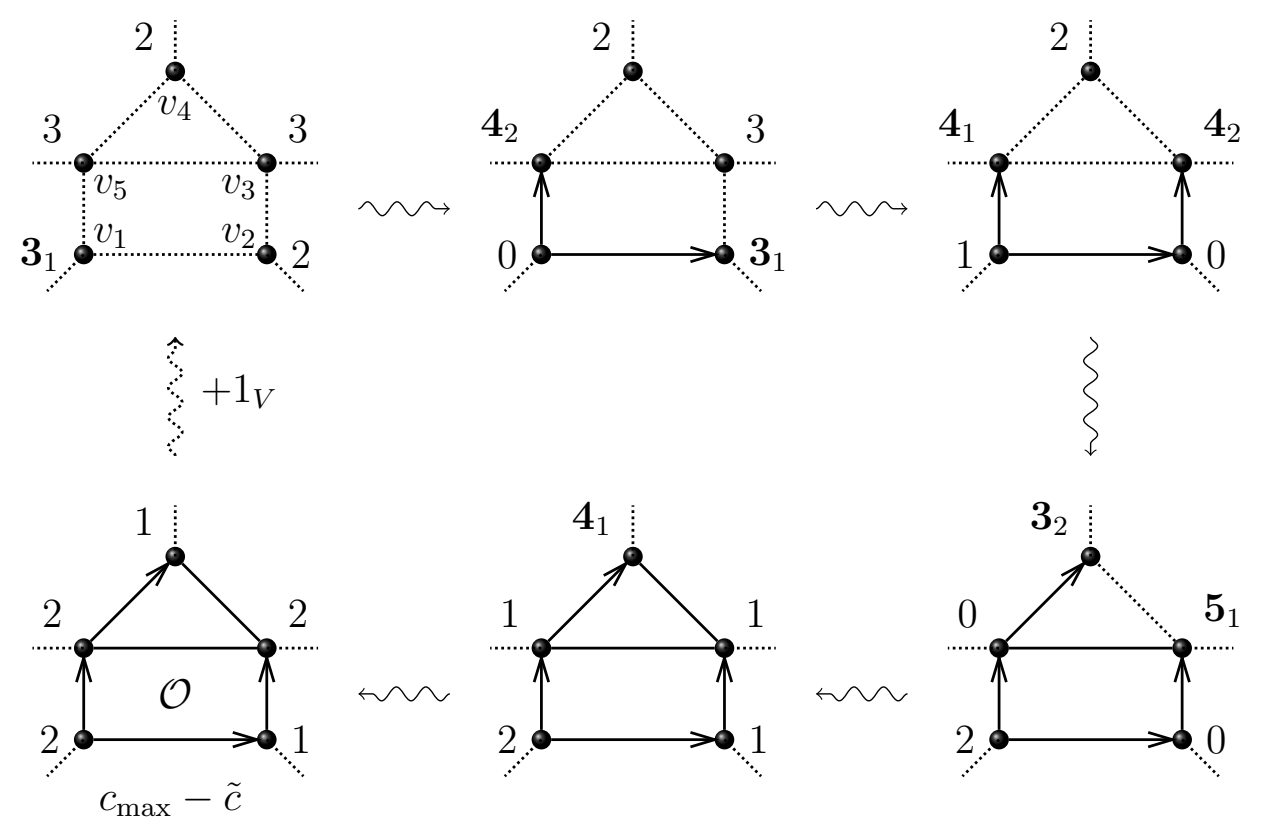

Figure 11: Superstables algorithm (cf. Example 18). Start in the upper-left corner. Bold numbers connote unstable vertices. Subscripts indicate firing precedence.

There are mappings

$$
\begin{aligned}
\theta: \mathbb{I} & \rightarrow \mathcal{S} \\
P & \mapsto \sum_{v \in V}\left(n_{P}(v)-1\right) v,
\end{aligned}
$$

and

$$
\begin{aligned}
\psi: \mathbb{O} & \rightarrow \mathcal{S} \\
\mathcal{O} & \mapsto \sum_{v \in V}\left(\operatorname{indeg}_{\mathcal{O}}(v)-1\right) v .
\end{aligned}
$$

Let $\pi: \mathcal{S} \times \mathfrak{S} \rightarrow \mathcal{S}$ be the first projection mapping, and let $\phi$ and $\eta$ be the mappings defined by the superstables algorithm.

The following diagram commutes:

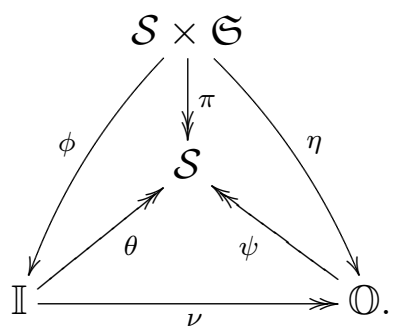

All mappings in the diagram are surjective except possibly for $\phi$ and $\eta$. 
Proof. The image of $\phi$ is in $\mathbb{I}$. In stabilizing $b$, each vertex eventually becomes unstable and is assigned an interval. Thus, the semiorder produced by the algorithm is a semiorder on all the vertices of $G$.

The image of $\theta$ is in $\mathcal{S}$. Let $P \in \mathbb{I}$. Then $P$ is isomorphic to the semiorder on a collection of intervals, $\{J(v): v \in V\}$. Letting $c=\theta(P)$, we must show that $c+1_{V}$ is a superstable configuration of $K(G)$. Let $c_{\max }$ be the maximal stable configuration on $K(G)$, and define $b=c_{\max }-\left(c+1_{V}\right)$. Note that $b \geqslant 0$. Starting with $b$ and firing the sink of $K(G)$ gives $\tilde{b}=c_{\max }-c$. By Propositions 7 and 8 , we must show that there is an ordering of $V$ forming a legal firing sequence for $\tilde{b}$.

Choose any $\sigma \in \mathfrak{S}$ so that $\sigma(i)<\sigma(j)$ if $\min J_{i}<\min J_{j}$. In particular, this means that if $v_{i}<v_{j}$ in $P$, then $\sigma(i)<\sigma(j)$. The $v$-th component of $\tilde{b}$ is

$$
\tilde{b}_{v}=\operatorname{deg}_{K(G)}(v)-n_{P}(v) .
$$

We can legally fire the vertices in the order given by $\sigma$ since when it becomes $v$ 's turn to fire, it will have received $n_{P}(v)$ grains of sand from the firings of those vertices $u$ neighboring $v$ such that $u<v$ and will thus be unstable.

We have $\theta \circ \phi=\pi$. Let $P=\phi(c, \sigma)$ and $b=c_{\max }-c$ where $c_{\max }$ is the maximal stable configuration on $K(G)$. Let $v \in V$. If $v$ is unstable in $b$, then $c_{v}=-1$ and there are no vertices smaller than $v$ in $P$. Hence, $n_{P}(v)-1=c_{v}=-1$, as required. Otherwise, in the course of the algorithm, say $u$ is the vertex whose firing causes $v$ to become unstable. Then the vertices that are less than $v$ in $P$ are exactly the vertices $w$ such that $w \leqslant u$. Among these $w$, only those that are attached to $v$ by an edge contribute to making $v$ unstable. Thus, exactly $n_{P}(v)$ grains of sand are added to $v$ in $b$ to make $v$ unstable. Since $b_{v}=\operatorname{deg}_{K(G)} v-1-c_{v}$, we have that $n_{P}(v)=1+c_{v}$, as required.

The mapping $\nu$ is surjective. The surjectivity of $\nu$ follows from Theorem 16.

We have $\psi \circ \nu=\theta$ and the image of $\psi$ is in $\mathcal{S}$. Let $P \in \mathbb{I}$ and $\mathcal{O}=\nu(P)$. Since $\mathcal{O}$ is compatible with $P$, we have that $n_{P}(v)=\operatorname{indeg}_{\mathcal{O}}(v)$ for each $v \in V$. Hence, $\psi(\nu(P))=\theta(P)$ as mappings of configurations. Since $\nu$ is surjective, the image of $\psi$ is contained in the image of $\theta$, hence in $\mathcal{S}$.

We have $\nu \circ \phi=\eta$. Given $(c, \sigma) \in \mathcal{S} \times \mathfrak{S}$, let $\mathcal{O}=\eta(c, \sigma)$ and $P=\phi(c, \sigma)$. We must show that $\mathcal{O}$ is compatible with $P$. Let $b$ be as in the algorithm. Run the algorithm up until it is a vertex $u$ 's turn to fire. Suppose $\{u, v\} \in E$. Then $v$ being stable at this point is equivalent to $u<v$ in $P$ and equivalent to $(u, v) \in \mathcal{O}$.

Corollary 20. Let $\sigma \in \mathfrak{S}$. Then the mappings

$$
\phi_{\sigma}: \mathcal{S} \rightarrow \mathbb{I}, \quad \eta_{\sigma}: \mathcal{S} \rightarrow \mathbb{O},
$$

defined by $\phi_{\sigma}(c)=\phi(c, \sigma)$ and $\eta_{\sigma}(c)=\eta(c, \sigma)$, are injective with left inverses $\theta$ and $\psi$, respectively.

Let $\mathcal{S}_{\max }$ denote the maximal quasi-superstables under the relation " $<$ " defined in Section 2.4, and let $\mathbb{O}_{\max }$ denote the acyclic orientations of $G$ (elements of $\mathbb{O}$ in which 
each edge is oriented). For each $c \in \mathcal{S}$, define the degree of $c$ to be $\operatorname{deg}(c):=\sum_{v \in V} c_{v}$. Then,

1. the restriction of $\eta_{\sigma}$ to $\mathcal{S}_{\max }$ gives a bijection

$$
\eta_{\sigma}: \mathcal{S}_{\max } \rightarrow \mathbb{O}_{\max }
$$

2. for $c \in \mathcal{S}$, we have $\operatorname{deg}(c) \leqslant g-1$, where $g:=|E|-|V|+1$ is the genus of $G$, with equality if and only if $c \in \mathcal{S}_{\max }$.

Proof. Both $\phi_{\sigma}$ and $\eta_{\sigma}$ are injective on $\mathcal{S}$ with the claimed left inverses since the restriction of $\pi$ in diagram 3 of Theorem 19 to $\mathcal{S} \times\{\sigma\}$ is bijective.

To show $\eta_{\sigma}\left(\mathcal{S}_{\text {max }}\right) \subseteq \mathbb{O}_{\text {max }}$, let $c \in \mathcal{S}$ and suppose that $\mathcal{O}:=\eta_{\sigma}(c) \notin \mathbb{O}_{\text {max }}$. According to Theorem 16 there is a point $t=\left(t_{0}, \ldots, t_{n}\right) \in \mathbb{R}^{n+1}$ and a corresponding $G$-semiorder, $P_{t}$, such that $\nu\left(P_{t}\right)=\mathcal{O}$. For ease of notation, we may assume that $t_{0} \leqslant \ldots \leqslant t_{n}$. Choose $t^{\prime} \in \mathbb{R}^{n+1}$ such that $t_{i}^{\prime} \geqslant t_{i}$ for all $i$ and $t_{i+1}^{\prime}>t_{i}^{\prime}+1$ for $0 \leqslant i \leqslant n-1$. Let $\mathcal{O}^{\prime}:=\nu\left(P_{t^{\prime}}\right)$ and $c^{\prime}:=\psi\left(\mathcal{O}^{\prime}\right)$. Then the set of oriented edges $\mathcal{O}$ is a proper subset of $\mathcal{O}^{\prime}$. Hence, $c<c^{\prime}$, showing $c \notin \mathcal{S}_{\max }$, as desired.

Now let $c \in \mathcal{S}$, and choose any $\mathcal{O} \in \mathbb{O}$ such that $c=\psi(\mathcal{O})$. From the definition of $\psi$, we have $\operatorname{deg}(c)=|\mathcal{O}|-|V| \leqslant|E|-|V|=g-1$, with equality exactly when $\mathcal{O} \in \mathbb{O}_{\text {max }}$. If $c<c^{\prime}$ for some quasi-superstable $c^{\prime}$, then since $\operatorname{deg}(c)<\operatorname{deg}\left(c^{\prime}\right)$, it follows that $\mathcal{O} \notin \mathbb{O}_{\max }$. This shows, for instance, that $\psi\left(\mathbb{O}_{\max }\right) \subseteq \mathcal{S}_{\max }$.

It remains to be shown that $\psi$ restricted to $\mathbb{O}_{\max }$ is injective. Let $c \in \mathcal{S}_{\max }$ and choose any $\mathcal{O}$ such that $c=\psi(\mathcal{O})$. We have seen that $\mathcal{O} \in \mathbb{O}_{\text {max }}$. From the definition of $\psi$, we see that indeg $\mathcal{O}_{\mathcal{O}}(v)$ is determined by $c$ for each $v \in V$. Then $\operatorname{outdeg}_{\mathcal{O}}(v)=\operatorname{deg}_{G}(v)-\operatorname{indeg}_{\mathcal{O}}$ since $\mathcal{O}$ orients every edge of $G$, and as noted in [3], these outdegrees determine $\mathcal{O}$. To see this, consider the graph $G$ oriented by $\mathcal{O}$. Since $\mathcal{O}$ is acyclic, this oriented graph must have sinks, i.e., vertices with outdegree 0 . Thus, $c$ determines the orientation of all edges incident on these sinks. Now remove these sinks and their incoming edges. The orientation of the resulting graph is still acyclic, and its sinks may also be determined from $c$. Iterate to see that $\mathcal{O}$ is determined by $c$, and hence, $\psi$ is injective when restricted to $\mathbb{O}_{\max }$.

Theorem 21. The mapping $\nu$ is bijective if and only if $G$ is a complete graph.

Proof. The surjectivity of $\nu$ is part of Theorem 19. Suppose that $G$ is a complete graph and that $\nu\left(P_{1}\right)=\nu\left(P_{2}\right)$ for some $P_{1}, P_{2} \in \mathbb{I}$. Then $v_{i}<v_{j}$ in $P_{1}$ implies $\left(v_{i}, v_{j}\right) \in \nu\left(P_{1}\right)$, which in turn implies $v_{i}<v_{j}$ in $P_{2}$. Similarly, $v_{i}<v_{j}$ in $P_{2}$ implies $v_{i}<v_{j}$ in $P_{1}$. Thus, $P_{1}=P_{2}$, so $\nu$ is injective.

Now suppose $G$ is not complete, i.e., there exist $v_{i}, v_{j} \in V$ such that $\left\{v_{i}, v_{j}\right\} \notin E$. Let $P_{1}$ be the $G$-semiorder where no vertices are comparable, and let $P_{2}$ be the $G$-semiorder where $v_{i}<v_{j}$ and all other pairs of vertices are incomparable. We have $\nu\left(P_{1}\right)=\emptyset=\nu\left(P_{2}\right)$. So $\nu$ is not injective. 


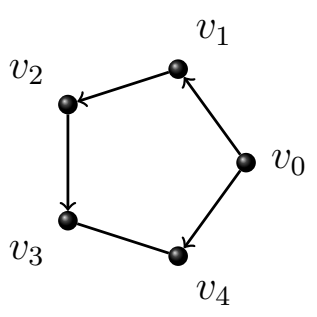

$\mathcal{O}_{P}$

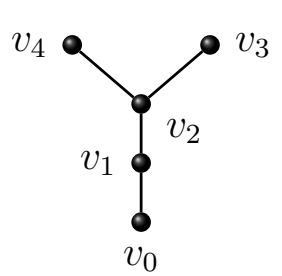

$P$

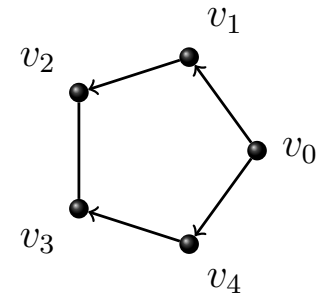

$\mathcal{O}^{\prime}$

Figure 12: Figure for Example 22.

Example 22. The mappings $\phi$ and $\eta$ are not necessarily surjective. Consider the case where $G$ is a cycle graph on five vertices. Figure 12 displays the Hasse diagram of a $G$-semiorder, $P$, and its compatible $G$-semiorientation $\nu(P)=\mathcal{O}_{P}$. For any ordering $\sigma$ of the vertices, $\eta\left(\psi\left(\mathcal{O}_{P}\right), \sigma\right)=\mathcal{O}^{\prime}$ where $\mathcal{O}^{\prime}$ is as pictured in Figure 12 .

\subsection{Labeling regions with superstables}

We can inductively label the regions of the $G$-semiorder arrangement, $\mathscr{I}$, with quasisuperstables. We call this labeling the (generalized) Pak-Stanley labeling after [16]. Start with the central region of $\mathscr{I}$, the region defined by $\left|x_{i}-x_{j}\right|<1$ for all $i, j$ such that $\left\{v_{i}, v_{j}\right\}$ is an edge of $G$. Label this region with the divisor $-1_{V}$. Put the central region in a queue, $Q$. Then, for as long as $Q$ is not empty:

1. Remove the first region $r$ from $Q$.

2. For each unlabeled region $r^{\prime}$ bordering $r$ :

(a) Determine the unique indices $i \neq j$ such that $\left|x_{i}-x_{j}\right|<1$ in $r$ but $x_{j}>x_{i}+1$ in $r^{\prime}$.

(b) If $r$ is labeled by $c=\sum_{k=0}^{n} c_{k} v_{k}$, then label $r^{\prime}$ by $c^{\prime}=c+v_{j}$.

(c) Add $r^{\prime}$ to the end of $Q$.

For each $r \in \mathcal{R}$, define $\lambda(r)$ to be the label assigned to $r$ by the above algorithm. Theorem 23 guarantees that when the algorithm terminates, the regions are labeled with quasi-superstables and the labels are, in fact, independent of the order in which regions are removed from the queue.

Recall the bijection $\rho: \mathbb{O} \rightarrow \mathcal{R}$, and let $\tau=\rho^{-1}$. Also recall the mapping $\psi$ from Theorem 19 and the mapping $\eta_{\sigma}$ from Corollary 20 .

Theorem 23. We have $\lambda=\psi \circ \tau$. So there is a surjective mapping

$$
\lambda: \mathcal{R} \longrightarrow \mathcal{S}
$$


and, for each $\sigma \in \mathfrak{S}$, a commutative diagram

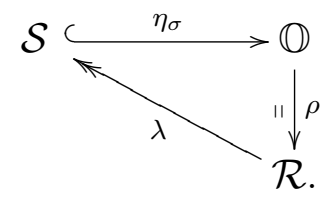

Proof. All the statements in the theorem follow directly from the equality $\lambda=\psi \circ \tau$, which we now prove by induction. If $r$ is the central region then $\tau(r)$ is the partial orientation in which all edges are marked blank. Therefore, in this case, $\lambda(r)=\psi(\tau(r))$.

Let the labeling algorithm run, and suppose that the region $r$ has just been removed from the queue. By induction, suppose that $\lambda=\psi \circ \tau$ when restricted to those regions that have been labeled so far. Let $r^{\prime}$ be an unlabeled region bordering $r$. Say that the inequalities that define $r$ are the same as those that define $r^{\prime}$ except that $\left|x_{i}-x_{j}\right|<1$ in $r$ and $x_{j}>x_{i}+1$ in $r^{\prime}$. It follows that if $\mathcal{O}=\tau(r)$ then $\tau\left(r^{\prime}\right)=\mathcal{O} \cup\left\{\left(v_{i}, v_{j}\right)\right\}$. Therefore, going from $\tau(r)$ to $\tau\left(r^{\prime}\right)$, only the indegree of $v_{j}$ has increased by one, so

$$
\begin{aligned}
\lambda\left(r^{\prime}\right) & =\lambda(r)+v_{j} \\
& =\psi(\tau(r))+v_{j} \\
& =\psi\left(\tau\left(r^{\prime}\right)\right) .
\end{aligned}
$$

The result follows by induction.

\section{$4 \quad$ Fixing a sink vertex}

In this section, we finally fully explain Figure 2. For that figure, we started with a graph with a given sink vertex and constructed a hyperplane arrangement with regions labeled by partial orientations of the graph and sandpile configurations. The nonnegative configurations arising were exactly the superstables for the graph.

Let $G$ be a graph with vertices $V=\left\{v_{0}, \ldots, v_{n}\right\}$, and designate vertex $v_{0}$ as the sink.

Definition 24. The $\left(G, v_{0}\right)$-semiorientations, denoted $\mathbb{O}_{0}$, are the $G$-semiorientations satisfying the additional requirement that $v_{0}$ is a source:

$$
\mathbb{O}_{0}=\left\{\mathcal{O} \in \mathbb{O}: \operatorname{outdeg}_{\mathcal{O}}\left(v_{0}\right)=\operatorname{deg}\left(v_{0}\right)\right\} .
$$

The set of admissible $\left(G, v_{0}\right)$-semiorientations is

$$
\widetilde{\mathbb{O}}_{0}=\left\{\mathcal{O} \in \mathbb{O}_{0}: \operatorname{indeg}_{\mathcal{O}}\left(v_{i}\right) \geqslant 1 \text { for all } i \neq 0\right\} .
$$

Thus, while $v_{0}$ is the sink for the sandpile model on $G$-i.e., the sink for the sake of defining the sandpile group, superstable configurations, and $G$-parking functions - it is a source for any $\mathcal{O} \in \mathbb{O}_{0}$.

Example 25. Figure 2 shows the admissible $(G, q)$-semiorientations for the graph in Figure 3. Due to lack of space, the requisite edges $\left(q, v_{1}\right)$ and $\left(q, v_{2}\right)$ are understood in Figure 2, but not drawn. 
Our next goal is to describe the image of $\mathbb{O}_{0}$ under the mapping $\psi: \mathbb{O} \rightarrow \mathcal{S}$ from Theorem 19, thus accounting for the configurations labeling the regions in Figure 2. We show below that the image of $\mathbb{O}_{0}$ is the set of quasi-superstables assigning the value -1 to $v_{0}$ and whose only other negative values must occur at vertices not connected to $v_{0}$ by an edge. Further, the image of $\widetilde{\mathbb{O}}_{0} \subseteq A_{0}$ is the set of $G$-parking functions of $G$.

Let $\widetilde{V}=V \backslash\left\{v_{0}\right\}$, and let

$$
X=\left\{v \in \widetilde{V}:\left\{v, v_{0}\right\} \notin E\right\} .
$$

Define $1_{X}=\sum_{v \in X} v$, a configuration on $G$ (having chosen $v_{0}$ as the sink). Let $K(G)_{0}$ denote the graph $G$ but with an edge $\left\{v, v_{0}\right\}$ added for each $v \in X$, and fix $v_{0}$ as its sink. Thus, configurations on $G$ and on $K(G)_{0}$ are elements of $\mathbb{Z} \widetilde{V}$, the free abelian group on $\widetilde{V}$, a subgroup of $\mathbb{Z} V$, the configurations on $K(G)$. Recall that $K(G)$ is the graph used to define quasi-superstables.

\section{Theorem 26.}

1. Define

$$
\mathcal{S}_{0}=\left\{c \in \mathcal{S}: c_{v_{0}}=-1 \text { and } c+v_{0} \geqslant-1_{X}\right\}
$$

Then

$$
\begin{aligned}
\psi\left(\mathbb{O}_{0}\right) & =\left\{\tilde{c}-v_{0}: \tilde{c}+1_{X} \text { a superstable on } K(G)_{0}\right\} \\
& =\mathcal{S}_{0}
\end{aligned}
$$

2. Define

$$
\widetilde{\mathcal{S}}_{0}=\left\{c \in \mathcal{S}: c_{v_{0}}=-1 \text { and } c+v_{0} \geqslant 0\right\} \subseteq \mathcal{S}_{0}
$$

Then

$$
\begin{aligned}
\psi\left(\widetilde{\mathbb{O}}_{0}\right) & =\left\{\tilde{c}-v_{0}: \tilde{c} \text { a superstable on } G\right\} \\
& =\left\{\tilde{c}-v_{0}: \tilde{c}+1_{X} \text { a superstable on } K(G)_{0} \text { and } \tilde{c} \geqslant 0\right\} \\
& =\widetilde{\mathcal{S}}_{0} .
\end{aligned}
$$

Thus, $\psi\left(\widetilde{\mathbb{O}}_{0}\right)=\widetilde{\mathcal{S}}_{0}$ is the set of $G$-parking functions with respect to $v_{0}$.

Proof. We first prove

Claim A: If $c \in \mathbb{Z} \widetilde{V}$, then $c+1_{X}$ is superstable on $K(G)_{0}$ if and only if $c-v_{0}+1_{V}$ is superstable on $K(G)$ and $c \geqslant-1_{X}$.

For any graph $H$, let $E_{H}$ denote its edges. Recall that $\tilde{q}$ is the sink vertex for $K(G)$. Let $c \in \mathbb{Z} \widetilde{V}$ with $c \geqslant-1_{X}$, and consider $c-v_{0}+1_{V}$ as a configuration on $K(G)$. Let $U \subseteq V$. If $v_{0} \in U$, then we cannot legally fire $U$ from $c-v_{0}+1_{V}$ : since $\left(c-v_{0}+1_{V}\right)_{v_{0}}=0$ and $\left\{v_{0}, \tilde{q}\right\} \in E_{K(G)}$, firing $U$ would result in a configuration with a negative $v_{0}$-component. 
Thus, $c-v_{0}+1_{V}$ is superstable if and only if there are no nonnempty subsets $U \subseteq \widetilde{V}$ that can be legally fired.

For $v \in U \subseteq \widetilde{V}$, consider the edges incident with $v$ that lead out of $U$ :

$$
\begin{aligned}
M(v, U) & =\left\{w \in(V \cup\{\tilde{q}\}) \backslash U:\{v, w\} \in E_{K(G)}\right\} \\
M(v, U)_{0} & =\left\{w \in V \backslash U:\{v, w\} \in E_{K(G)_{0}}\right\} .
\end{aligned}
$$

Then,

$$
M(v, U) \backslash M(v, U)_{0}=\{\tilde{q}\} \quad \text { and } \quad M(v, U)_{0} \backslash M(v, U)= \begin{cases}\emptyset & \text { if } v \notin X \\ \left\{v_{0}\right\} & \text { if } v \in X\end{cases}
$$

So the cardinality of $M(v, U)$ is

$$
|M(v, U)|= \begin{cases}\left|M(v, U)_{0}\right|+1 & \text { if } v \notin X \\ \left|M(v, U)_{0}\right| & \text { if } v \in X\end{cases}
$$

Now $c-v_{0}+1_{V}$ is superstable on $K(G)$ if and only if $\left(c-v_{0}+1_{V}\right)_{v}<|M(v, U)|$ for all $v \in U$ for all nonempty $U \subseteq \widetilde{V}$, and $c+1_{X}$ is superstable on $K(G)_{0}$ if and only if $\left(c+1_{X}\right)_{v}<\left|M(v, U)_{0}\right|$ for all $v \in U$ for all nonempty $U \subseteq \widetilde{V}$. For $v \in \widetilde{V}$ we have

$$
\left(c-v_{0}+1_{V}\right)_{v}=c_{v}+1= \begin{cases}\left(c+1_{X}\right)_{v}+1 & \text { if } v \notin X \\ \left(c+1_{X}\right)_{v} & \text { if } v \in X .\end{cases}
$$

So Claim A follows from (5). The condition $c \geqslant-1_{X}$ is required in the statement of the claim since superstables must be nonnegative.

Now let

$$
P=\left\{\tilde{c}-v_{0}: \tilde{c}+1_{X} \text { superstable on } K(G)_{0}\right\} .
$$

The fact that $P=\mathcal{S}_{0}$ follows directly from Claim A. We now show that $\psi\left(\mathbb{O}_{0}\right)=\mathcal{S}_{0}$ to finish the proof of part (1). Let $\mathcal{O} \in \mathbb{O}_{0}$. Then $\psi(\mathcal{O}) \in \mathcal{S}$ by Theorem 19. Since $v_{0}$ is a source for $\mathcal{O}$, we have $\psi(\mathcal{O})_{v_{0}}=-1$, and if $v \in \widetilde{V} \backslash X$, then $\psi(\mathcal{O})_{v} \geqslant 0$. Thus, $\psi(\mathcal{O}) \in \mathcal{S}_{0}$. Conversely, given $c \in \mathcal{S}_{0}$, run the superstables algorithm from Section 3.3 with any vertex ordering of $V$ in which $v_{0}$ appears first. Using the notation from the initialization stage of the algorithm, let $b=c_{\max }-c$. Since $c_{v_{0}}=-1$, the vertex $v_{0}$ is unstable in $b$ and will fire first. Since $c+v_{0} \geqslant-1_{X}$, no vertex $v \in \widetilde{V} \backslash X$ is unstable in $b$. So when $v_{0}$ fires, the algorithm will orient each edge incident on $v_{0}$ out from $v_{0}$. If $\mathcal{O}$ is the semiorientation produced by the algorithm, it follows that $\mathcal{O} \in \mathbb{O}_{0}$ and, by Theorem 19, we have $\psi(\mathcal{O})=c$. Thus, $\psi\left(\mathbb{O}_{0}\right)=\mathcal{S}_{0}$.

To prove part (2), let

$$
\begin{aligned}
N & =\left\{\tilde{c}-v_{0}: \tilde{c} \text { a superstable on } G\right\}, \\
\widetilde{P} & =\left\{\tilde{c}-v_{0}: \tilde{c}+1_{X} \text { a superstable on } K(G)_{0} \text { and } \tilde{c} \geqslant 0\right\} \subseteq P .
\end{aligned}
$$


From part (1), it follows directly that $\psi\left(\widetilde{\mathbb{O}}_{0}\right)=\widetilde{P}=\widetilde{\mathcal{S}}_{0}$. To show $N=\widetilde{\mathcal{S}}_{0}$ and finish, proceed exactly as in the proof of Claim A. Let $\tilde{c} \in \mathbb{Z} \widetilde{V}$ with $\tilde{c} \geqslant 0$. We must show that $\tilde{c}$ is superstable on $G$ if and only if $\tilde{c}-v_{0}+1_{V}$ is superstable on $K(G)$. Given $v \in U \subset \widetilde{V}$, this time consider the set

$$
M(v, U)_{G}=\left\{w \in V \backslash U:\{v, w\} \in E_{G}\right\},
$$

and note that $|M(v, U)|=\left|M(v, U)_{G}\right|+1$, with $M(v, U)$ defined as before, from which the result follows.

Part 2 of the following corollary recaptures a well-known result from sandpile theory. For instance, it occurs as Lemma 5 in [4] as a statement about recurrent configurations, equivalent to our statement in light of Proposition 7.

Corollary 27. Order the superstable configurations on $G$ by the relation " $<$ " defined in Section 2.4. Let $\mathcal{S}_{\max }$ denote the maximal quasi-superstables on $G$, and let $g:=|E|-|V|-1$ be the genus of $G$, as in Corollary 20. Let $\tilde{c}$ be a superstable configuration on $G$. Then,

1. $\tilde{c}$ is maximal if and only if $\tilde{c}-v_{0} \in \widetilde{\mathcal{S}}_{0} \cap \mathcal{S}_{\max }$,

2. $\operatorname{deg}(\tilde{c}):=\sum_{v \in \widetilde{V}} \tilde{c}_{v} \leqslant g$ with equality if and only if $\tilde{c}$ is maximal.

Proof. By Theorem $26(2), \tilde{c}-v_{0} \in \widetilde{\mathcal{S}}_{0}$. Hence, part (2) follows immediately from part (1) by Corollary $20(2)$. We now prove part (1).

$(\Rightarrow)$ Suppose $\tilde{c}-v_{0} \notin \mathcal{S}_{\text {max }}$. By Theorem $26(2)$, there exists $\mathcal{O} \in \widetilde{\mathbb{O}}_{0}$ such that $\psi(\mathcal{O})=$ $\tilde{c}-v_{0}$. By Corollary 20, we have $\mathcal{O} \notin \mathbb{O}_{\max }$, i.e., $\mathcal{O}$ is not an acyclic orientation of $G$, but by the beginning of the proof to Corollary 20, there exists $\mathcal{O}^{\prime} \in \mathbb{O}_{\text {max }}$ such that $\mathcal{O}$ is a proper subset of $\mathcal{O}^{\prime}$. It follows that $\psi(\mathcal{O})=\tilde{c}-v_{0}<\psi\left(\mathcal{O}^{\prime}\right)=: c^{\prime}$, where $c^{\prime} \in \mathcal{S}$. Since $\mathcal{O} \subset \mathcal{O}^{\prime}$ and $\mathcal{O}^{\prime}$ is acyclic, we have $c_{v_{0}}^{\prime}=-1$ and $c^{\prime}+v_{0} \geqslant 0$, i.e., $c^{\prime} \in \widetilde{\mathcal{S}}_{0}$. Define $\tilde{c}^{\prime}$ by $c^{\prime}=\tilde{c}^{\prime}-v_{0}$. Then $\tilde{c}^{\prime}$ is a superstable configuration on $G$ by Theorem 26 , and $\tilde{c}<\tilde{c}^{\prime}$. So $\tilde{c}$ is not a maximal superstable.

$(\Leftarrow)$ Conversely, suppose $\tilde{c}-v_{0} \in \mathcal{S}_{\max }$. Take any superstable configuration $\tilde{c}^{\prime}$ such that $\tilde{c} \leqslant \tilde{c}^{\prime}$. Then $\tilde{c}^{\prime}-v_{0} \in \mathcal{S}$ by Theorem $26(2)$, and $\tilde{c}-v_{0} \leqslant \tilde{c}^{\prime}-v_{0}$. By maximality of $\tilde{c}-v_{0}$, it follows that $\tilde{c}=\tilde{c}^{\prime}$. Hence, $\tilde{c}$ is a maximal superstable.

Definition 28. The $\left(G, v_{0}\right)$-semiorder arrangement, denoted $\mathscr{I}_{0}$, is the set of hyperplanes in $\mathbb{R}^{n}$ given by

$$
x_{i}-x_{j}=1,
$$

for all $i, j$ not equal to 0 such that $\left\{v_{i}, v_{j}\right\} \in E$.

Definition 29. The regions of $\mathscr{I}_{0}$, denoted $\mathcal{R}_{0}$, are the connected components of $\mathbb{R}^{n} \backslash \mathscr{I}_{0}$.

Define the subset

$$
T_{0}=\left\{\left(x_{0}, \ldots, x_{n}\right) \in \mathbb{R}^{n+1}: x_{i}>x_{0}+1 \text { whenever }\left\{v_{i}, v_{0}\right\} \in E_{G}\right\}
$$


and let

$$
\mathcal{R}_{0}^{\prime}=\left\{r \in \mathcal{R}: r \subseteq T_{0}\right\} .
$$

The elements of $\mathcal{R}_{0}^{\prime}$ are exactly those regions with corresponding semiorientations (under $\rho$ ) having $v_{0}$ as a source. Hence, the bijection $\rho: \mathbb{O} \rightarrow \mathcal{R}$ restricts to a bijection $\mathbb{O}_{0} \rightarrow \mathcal{R}_{0}^{\prime}$. The projection mapping $\left(x_{0}, \ldots, x_{n}\right) \rightarrow\left(x_{1}, \ldots, x_{n}\right)$, omitting the 0 -th coordinate, induces a bijection

$$
\pi_{0}: \mathcal{R}_{0}^{\prime} \rightarrow \mathcal{R}_{0}
$$

Therefore, we have the following theorem.

Theorem 30. The mapping

$$
\rho_{0}:=\pi_{0} \circ \rho: \mathbb{O}_{0} \rightarrow \mathcal{R}_{0}
$$

is a bijection.

Example 31. The $\left(G, v_{0}\right)$-semiorder arrangement for graph $G$ of Figure 3 with $v_{0}=q$ is drawn in Figure 2. Its regions, $\mathcal{R}_{0}$, are the projections of the regions $\mathcal{R}_{0}^{\prime}$ displayed in Figure 6 .

The central region of $\mathscr{I}_{0}$ is the region defined by $\left|x_{i}-x_{j}\right|<1$ for all distinct $i, j$ not equal to 0 such that $\left\{v_{i}, v_{j}\right\}$ is an edge of $G$. Inductively label the regions of $\mathscr{I}_{0}$ as in Section (3.4), but starting with the central region labeled with the configuration that assigns 0 to all $v_{i}$ such that $\left\{v_{i}, v_{0}\right\} \in E$ and -1 to all other vertices, including $v_{0}$. For each $r \in \mathcal{R}_{0}$, define $\lambda_{0}(r)$ to be the label assigned to $r$ in this fashion.

Define $\tau_{0}=\rho_{0}^{-1}$. There is a version of Theorem 3.4 in this context (proved similarly):

Theorem 32. We have $\lambda_{0}=\psi \circ \tau_{0}$. So there is a surjective mapping

$$
\lambda_{0}: \mathcal{R}_{0} \longrightarrow \mathcal{S}_{0}
$$

and, for each $\sigma \in \mathfrak{S}_{0}$, a commutative diagram

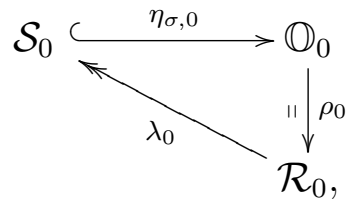

where $\eta_{\sigma, 0}$ is the restriction of $\eta_{\sigma}$ to $\mathcal{S}_{0}$.

For $i=1, \ldots, n$, if $\left\{v_{i}, v_{0}\right\} \in E_{G}$, let

$$
\mathbb{R}_{(i, 0)}^{n}=\mathbb{R}^{n}
$$

otherwise, if $\left\{v_{i}, v_{0}\right\} \notin E_{G}$, let

$$
\mathbb{R}_{(i, 0)}^{n}=\left\{\left(x_{1}, \ldots, x_{n}\right) \in \mathbb{R}^{n}: x_{i}>x_{j}+1 \text { for some } j \text { with }\left\{v_{i}, v_{j}\right\} \in E\right\} \text {. }
$$

Define $\widetilde{\mathbb{R}}^{n}=\bigcap_{i=1}^{n} \mathbb{R}_{(i, 0)}^{n}$. 
Definition 33. The admissible regions of $\mathscr{I}_{0}$, denoted $\widetilde{\mathcal{R}}_{0}$, are the connected components of $\widetilde{\mathbb{R}}^{n} \backslash \mathscr{I}_{0}$.

Note that $\widetilde{\mathcal{R}}_{0}=\mathcal{R}_{0}$ exactly when each nonsink vertex is connected by an edge to the sink.

The admissible regions of $\mathscr{I}_{0}$ are exactly those regions whose corresponding $G$-semiorientations satisfy $\operatorname{indeg}_{\mathcal{O}}\left(v_{i}\right) \geqslant 1$ for all $i \neq 0$. So combining Theorem 26 and Theorem 32 gives

\section{Theorem 34 .}

$$
\lambda_{0}\left(\widetilde{\mathcal{R}}_{0}\right)=\widetilde{\mathcal{S}}_{0}=\left\{c-v_{0}: \text { c a superstable on } G\right\}
$$

Example 35. The 9 admissible regions in Figure 2 are labeled by the 8 distinct superstables (or $G$-parking functions if one remembers that the sink is labeled by -1 ). The zero-configuration appears twice.

\section{Conclusion.}

Let $A$ be an $(n+1) \times(n+1)$ matrix. Define $\mathcal{H}_{A}$ to be the set of hyperplanes

$$
x_{i}-x_{j}=A_{i j}
$$

for all $i \neq j$ such that $\left\{v_{i}, v_{j}\right\} \in E$. For example, $\mathcal{H}_{A}=\mathscr{I}$ if $A$ has all 1 s as its entries.

Define the regions of $\mathcal{H}_{A}$, denoted $\mathcal{R}_{A}$, to be the connected components of $\mathbb{R}^{n+1} \backslash \mathcal{H}_{A}$. The set of inequalities $x_{i}-x_{j}<A_{i j}$ for all $i$ and $j$ defines the central region of $\mathcal{H}_{A}$. We say that $\mathcal{H}_{A}$ has a central region if this central region is nonempty.

Conjecture 36. Suppose that $\mathcal{H}_{A}$ has a central region. Labeling the regions of $\mathcal{H}_{A}$ as in Section 3.4 defines a surjection

$$
\lambda_{A}: \mathcal{R}_{A} \longrightarrow \mathcal{S}
$$

A similar conjecture holds if one first chooses $v_{0}$ as a sink: replace $A$ with an $n \times n$ matrix, and label regions as in Section 4. So the central region would be labeled with the configuration that assigns 0 to vertices connected to $v_{0}$ and -1 to the other vertices (including $v_{0}$ ). We conjecture that the nonnegative configurations that arise as labels are exactly the $G$-parking functions. The $G$-Shi conjecture of Duval, Klivans, and Martin is a special case.

In the spirit of [1] and [16], it would be interesting to extend our results to the case of multigraphs: graphs in which multiple edges are allowed between vertices. If there are $k$ edges between $v_{i}$ and $v_{j}$, one might replace the two hyperplanes $x_{i}-x_{j}= \pm 1$ with the $2 k$ hyperplanes $x_{i}-x_{j}= \pm 1, \ldots, \pm k$. 


\section{References}

[1] Christos A. Athanasiadis and Svante Linusson. A simple bijection for the regions of the Shi arrangement of hyperplanes. Discrete Math., 204(1-3):27-39, 1999.

[2] Per Bak, Chao Tang, and Kurt Wiesenfeld. Self-organized criticality: An explanation of $1 / f$ noise. Phys. Rev. Lett., 59(4):381-384, 1987.

[3] Brian Benson, Deeparnab Chakrabarty, and Prasad Tetali. G-parking functions, acyclic orientations and spanning trees. Discrete Math., 310(8):1340-1353, 2010.

[4] Norman Biggs. The Tutte polynomial as a growth function. J. Algebraic Combin., 10(2):115-133, 1999.

[5] Deepak Dhar. Self-organized critical state of sandpile automaton models. Phys. Rev. Lett., 64(14):1613-1616, 1990.

[6] Deepak Dhar. Theoretical studies of self-organized criticality. Phys. A, 369(1):29-70, 2006.

[7] Art Duval, Caroline Klivans, and Jeremy Martin. The G-shi arrangement, and its relation to $G$-parking functions. http://www.math.utep.edu/Faculty/duval/ papers/nola.pdf, January 2011.

[8] Eric Goles and Erich Prisner. Source reversal and chip firing on graphs. Theoret. Comput. Sci., 233(1-2):287-295, 2000.

[9] Curtis Greene and Thomas Zaslavsky. On the interpretation of Whitney numbers through arrangements of hyperplanes, zonotopes, non-Radon partitions, and orientations of graphs. Trans. Amer. Math. Soc., 280(1):97-126, 1983.

[10] Alexander E. Holroyd, Lionel Levine, Karola Mészáros, Yuval Peres, James Propp, and David B. Wilson. Chip-firing and rotor-routing on directed graphs. In In and out of equilibrium. 2, volume 60 of Progr. Probab., pages 331-364. Birkhäuser, Basel, 2008.

[11] Donald E. Knuth. The art of computer programming. Volume 3. Addison-Wesley Publishing Co., Reading, Mass.-London-Don Mills, Ont., 1973. Sorting and searching, Addison-Wesley Series in Computer Science and Information Processing.

[12] Lionel Levine and James Propp. What is ... a sandpile? Notices Amer. Math. Soc., 57(8):976-979, 2010.

[13] David Perkinson, Jacob Perlman, and John Wilmes. Primer for the algebraic geometry of sandpiles. arXiv:1112.6163, 2012.

[14] Alexander Postnikov and Boris Shapiro. Trees, parking functions, syzygies, and deformations of monomial ideals. Trans. Amer. Math. Soc., 356(8):3109-3142 (electronic), 2004.

[15] Jian Yi Shi. The Kazhdan-Lusztig cells in certain affine Weyl groups, volume 1179 of Lecture Notes in Mathematics. Springer-Verlag, Berlin, 1986. 
[16] Richard P. Stanley. Hyperplane arrangements, parking functions and tree inversions. In Mathematical essays in honor of Gian-Carlo Rota (Cambridge, MA, 1996), volume 161 of Progr. Math., pages 359-375. Birkhäuser Boston, Boston, MA, 1998.

[17] Richard P. Stanley. An introduction to hyperplane arrangements. In Geometric combinatorics, volume 13 of IAS/Park City Math. Ser., pages 389-496. Amer. Math. Soc., Providence, RI, 2007. 